\title{
Simulation of three-dimensional nonlinear water waves using a pseudospectral volumetric method with an artificial boundary condition
}

\author{
Klahn, Mathias; Madsen, Per A.; Fuhrman, David R.
}

Published in:

International Journal for Numerical Methods in Fluids

Link to article, DOI:

$10.1002 /$ fld.4956

Publication date:

2021

Document Version

Peer reviewed version

Link back to DTU Orbit

Citation $(A P A)$ :

Klahn, M., Madsen, P. A., \& Fuhrman, D. R. (2021). Simulation of three-dimensional nonlinear water waves using a pseudospectral volumetric method with an artificial boundary condition. International Journal for Numerical Methods in Fluids, 93(6), 1843-1870. https://doi.org/10.1002/fld.4956

\section{General rights}

Copyright and moral rights for the publications made accessible in the public portal are retained by the authors and/or other copyright owners and it is a condition of accessing publications that users recognise and abide by the legal requirements associated with these rights.

- Users may download and print one copy of any publication from the public portal for the purpose of private study or research.

- You may not further distribute the material or use it for any profit-making activity or commercial gain

- You may freely distribute the URL identifying the publication in the public portal 


\title{
Simulation of three-dimensional nonlinear water waves using a pseudospectral volumetric method with an artificial boundary condition
}

\author{
Mathias Klahn,1 Per A. Madsen ${ }^{1}$, and David R. Fuhrman ${ }^{1}$ \\ ${ }^{1}$ Department of Mechanical Engineering, Technical University of Denmark, Kgs. Lyngby, Denmark
}

(1) bstract

This paper presents a pseudospectral method for the simulation of nonlinear water waves described by potential flow theory in three spatial dimensions. The method utilizes an artificial boundary condition that limits the vertical extent of the fluid domain, and it is found that the reduction in domain size offered by the boundary condition enables the plution of the Laplace problem with roughly half the degrees of freedom compared to another spectral method in the literature for (wave number times water depth) $k h \geq 2 \pi$. Moreover, it is found that the location of the artificial oundary condition can be chosen once and for all at the beginning of simulations such that the size of the fluid Jomain is reduced substantially, even when the lowest point of the free surface elevation varies significantly with time. 1 'he method is tested by simulating steady nonlinear wave trains, the development of crescent waves from a steady nonlinear wave train, a nonlinear focusing event and a Gaussian hump which is initially at rest. It is shown that in all out the most nonlinear cases the method is capable of obtaining accurate results.

\section{Keywords:}

Yonlinear water waves, Spectral methods, Potential flow, Artificial boundary condition, Accuracy, Stability.

\section{Introduction}

their 1996-review, Tsai \& Yue [1] concluded that the numerical methods for simulation of nonlinear potential flow problems at that time were reasonably mature, although large problems were still out of reach due to lack of bbustness and efficiency of the methods. While intense research efforts and massive hardware improvements certainly ave advanced the numerical simulations quite significantly since then, the search for robust and efficient methods continues. Many of the currently available nonlinear free surface potential flow solvers suffer from unstable time Integration and combat this problem using artificial damping. The typical picture is that for waves whose degree of nonlinearity is not too large, artificial damping may be used to obtain a stable and accurate time integration. For s) eep waves this strategy, however, fails to deliver satisfying results. With that being said, the different methods ucm itely do vary in their robustness. Among the more robust methods we find the volumetric methods which map

the fluid domain to a time-independent rectangular box through a stretching of the vertical coordinate and solve the ansformed Laplace problem by discretizing the entire box. The research into these methods presumably began with the work of Li \& Fleming [2], who developed a finite difference-based multigrid method for three-dimensional wave

Pelds, and since then, many different authors have used a volumetric approach for wave simulations. For example, Singham \& Zhang [3], Engsig-Karup et al. [4], Christiansen et al. [5], Yates \& Benoit [8], Raoult et al. [7] and Klahn et al. [10] have all employed volumetric methods, and as a consequence it is now well established that this approach in handle variable bathymetry without approximations and that it leads to a stable computation of surface velocities as well as velocity profiles. These attractive features come at the prize of a substantially more involved computational 'rocedure than, for example, that of the classic High-Order Spectral method derived independently by Dommermuth \& Yue [14] and West et al. [15]. Although the HOS method is not capable of dealing with very steep waves (see e.g. Klahn et al. [11] for an investigation of its range of applicability), it is as a starting point much more efficient than the volumetric methods when the degree of nonlinearity is not too large. In fact, Ducrozet et al. [16] have shown that their implementation of the HOS method has an execution time which is typically two orders of magnitude smaller

${ }^{*}$ Corresponding author. E-mail: matkla@mek.dtu.dk

This article has been accepted for publication and undergone full peer review but has not been through the copyediting, typesetting, pagination and proofreading process which may lead to differences between this version and the Version of Record. Please cite this article as doi: 10.1002/fld.4956 
than that of the finite difference method of Ensig-Karup et al. [4] when dealing with long-time propagation of regular wave trains and requiring the final phase error to be less than 0.1 degrees (see Figure 13 of Ducrozet et al.).

For that reason, efforts have been made in order to increase the efficiency of the volumetric methods. Engsig-Karup et al. [17] have, for example, been able to increase the computational performance of their method substantially by executing it in parallel on a graphics processing unit. Another interesting attempt has been made by Nicholls [12], who showed that an artificial boundary condition could be used to reduce the vertical extent of the domain on which the Laplace equation must be solved. As a consequence, the boundary condition enabled the computation of the surface velocities to a given accuracy with fewer degrees of freedom in the vertical direction than if the whole fluid domain was discretized, giving rise to large computational savings. Nicholls did not test this strategy on any "real" ave problem, however, but instead used the method of manufactured solutions to test the solution of a single, time independent Laplace problem with (wave number times water depth) $k h=1000$. As such at least two different aspects of the boundary condition remain to be clarified. Since the effect on water waves by a change in the water depth is practically insignificant if $k h>2 \pi$ because the velocity is expected to decrease exponentially with depth in that case see e.g. the proof of this fact for two-dimensional wave fields given by Longuet-Higgins [18]), simulations with finite ater depth are usually carried out with $k h \leq 2 \pi$. It is hence interesting to know if noteworthy savings in the vertical resolution can also be made for these water depths. Moreover, it is interesting to know if it is possible in practice to choose the vertical location of the artificial boundary condition once and for all at the beginning of a simulation, such flyat at all times 1) it limits the vertical extent of the fluid domain substantially and 2) the lowest point of the free surface remains above the location of the boundary condition. Here the interest is motivated by the fact that several cbmputational quantities, which we will mention explicitly at a later stage, must be recomputed if the location of the boundary condition is changed during a simulation. While a good position for the boundary is easily chosen a priori in he case of steady waves, the positioning of the boundary is much harder when simulating irregular wave fields, since their lowest trough varies with time.

The aim of this work is to shed light on the above mentioned aspects of the artificial boundary condition when sing a psudospectral method in three spatial dimensions. To be specific, the goal of the investigation is to find out whether the location of the artificial boundary condition can be chosen such that it meets the requirements 1) and 2) above and how large computational savings can potentially be realized under these conditions. Along the way, we test me accuracy, stability and efficiency of the method. The method that we will describe assumes periodic boundary onditions in the two horizontal directions and employs the Fourier collocation method and the classical fourth order Runge-Kutta method to discretize the free surface boundary conditions in space and time, respectively. Moreover, the Slution of the Laplace problem is based on the artificial boundary condition used by Nicholls, in combination with a iscretization using the Fourier collocation method in the horizontal directions and the Legendre collocation method in the vertical direction.

The remainder of this paper is organized as follows: Section 2 gives an introduction to the physical system conidered, assumptions made and governing equations. Section 3 gives a detailed description of the numerical method, yhile Sections 4-7 present the results that we have obtained using it to simulate steady nonlinear waves, the formaTom of crescent waves, a nonlinear focusing event and a Gaussian hump initially at rest. In section 8 we discuss the
omputational efficiency of the numerical method before conclusions are finally drawn in Section 9 .

\section{Physical system and governing equations}

We consider the motion of an inviscid, incompressible and irrotational fluid with a free surface in three dimensions $(x$, and $z$ ) and assume the motion to be spatially periodic in the horizontal $x$ and $y$ directions with periods $L_{x}$ and $L_{y}$, espectively. In the vertical $z$ direction, the fluid is taken to be bounded from below by an impermeable flat seabed at $z=-h$, to have its still water level at $z=0$ and to be bounded from above by its free surface $\eta(x, y, t)$. We note here

lat this way of representing the free surface limits our description to non-overturning waves.

For this physical system, the equations of motion are the irrotational Euler equations, which take their simplest form when written in terms of the velocity potential $\Phi$. As Zakharov [13] proved now more than 50 years ago, the state of the system is uniquely determined by the free surface elevation and the velocity potential at the free surface, 
$\Phi_{s}=\left.\Phi\right|_{z=\eta}$, and we follow his work and write the governing equations as

$$
\begin{array}{lr}
\frac{\partial^{2} \Phi}{\partial x^{2}}+\frac{\partial^{2} \Phi}{\partial y^{2}}+\frac{\partial^{2} \Phi}{\partial z^{2}}=0 & \text { for }-h<z<\eta(x, y, t) \\
\frac{\partial \Phi}{\partial z}=0 & \text { for } z=-h, \\
\frac{\partial \eta}{\partial t}=\left(1+\left(\frac{\partial \eta}{\partial x}\right)^{2}+\left(\frac{\partial \eta}{\partial y}\right)^{2}\right) w_{s}-\frac{\partial \eta}{\partial x} \frac{\partial \Phi_{s}}{\partial x}-\frac{\partial \eta}{\partial y} \frac{\partial \Phi_{s}}{\partial y}, & \\
\frac{\partial \Phi_{s}}{\partial t}=-g \eta-\frac{1}{2}\left(\left(\frac{\partial \Phi_{s}}{\partial x}\right)^{2}+\left(\frac{\partial \Phi_{s}}{\partial y}\right)^{2}\right)+\frac{1}{2}\left(1+\left(\frac{\partial \eta}{\partial x}\right)^{2}+\left(\frac{\partial \eta}{\partial y}\right)^{2}\right) w_{s}^{2}, &
\end{array}
$$

where $w_{s}=\left.\partial_{z} \Phi\right|_{z=\eta}$ is the vertical velocity of the fluid at the free surface and $g$ denotes the gravitational acceleration fr which we shall use the value $9.81 \mathrm{~m} / \mathrm{s}^{2}$ throughout this work. The physical meaning of these equations is that he Laplace equation (1a) expresses conservation of mass, while the boundary condition (1b) states that the seabed is innermeable. Moreover, the kinematic boundary condition (1c) follows from the fact that a fluid particle cannot pass through the free surface, and the dynamic boundary condition (1d) states that the pressure along the free surface is onstant.

\section{Numerical methods}

This section is devoted to the description of the numerical method that we employ to integrate (1) in time. This system of equations constitutes an initial value problem for the quantities $\eta$ and $\Phi_{s}$ in the sense that if $\eta$ and $\Phi_{s}$ are litially known, then $w_{s}$ can be computed from the Laplace problem consisting of (1a) and (1b), supplemented with the boundary condition $\left.\Phi\right|_{z=\eta}=\Phi_{s} ; \eta$ and $\Phi_{s}$ can then in turn be stepped forward in time using (1c) and (1d). Due to this structure of the problem, we will in Section 3.1 describe how the boundary conditions at the free surface are tegrated in time under the assumption that $w_{s}$ is known, before we present the method with which we compute $w_{s}$ f om $\eta$ and $\Phi_{s}$ in Section 3.2. In both parts of the method we seek approximations of the relevant quantities in the form of series expansions, for the simple reason that for non-overturning waves these quantities will always be smooth inctions of $x, y$ and possibly $z$. As such, the series representations will converge at a rate faster than any algebraic ate (see e.g. Kopriva [21]), meaning that only few degrees of freedom are needed to obtain accurate approximations. Moreover, we want to emphasize that neither of the two parts employ any kind of dealiasing. We have made this choice as we have found that dealiasing substantially decreases the method's computational performance both in terms of computation time and memory requirements while seeming to give no improvement of the method in terms of accuracy and stability.

\section{(1) Time integration of the free surface boundary conditions}

To integrate the free surface boundary conditions in time we employ a method of lines approach and discretize the

P patial and temporal dimensions separately. Owing to the periodicity assumptions in the $x$ and $y$ directions, we iscretize the spatial part of equations (1c) and (1d) using the Fourier collocation method. By this we mean that we satisfy (1c) and (1d) at the set of grid points

$$
\left\{\left(x_{n_{x}}, y_{n_{y}}\right)=\left(\frac{L_{x} n_{x}}{2 N_{x}}, \frac{L_{y} n_{y}}{2 N_{y}}\right) \mid 0 \leq n_{x}<2 N_{x} \text { and } 0 \leq n_{y}<2 N_{y}\right\}
$$

with the underlying assumption that the functions $\eta, \Phi_{s}$ and $w_{s}$ are given by the truncated Fourier series

$$
\begin{aligned}
& \eta(x, y, t)=\sum_{n_{x}=-N_{x}}^{N_{x}-1} \sum_{n_{y}=-N_{y}}^{N_{y}-1} \widehat{\eta}_{n_{x}, n_{y}} \exp \left(i \mathbf{k}_{n_{x}, n_{y}} \cdot \mathbf{r}\right) \\
& \Phi_{s}(x, y, t)=\sum_{n_{x}=-N_{x}}^{N_{x}-1} \sum_{n_{y}=-N_{y}}^{N_{y}-1} \widehat{\Phi}_{n_{x}, n_{y}} \exp \left(i \mathbf{k}_{n_{x}, n_{y}} \cdot \mathbf{r}\right) \\
& w_{s}(x, y, t)=\sum_{n_{x}=-N_{x}}^{N_{x}-1} \sum_{n_{y}=-N_{y}}^{N_{y}-1} \widehat{w}_{n_{x}, n_{y}} \exp \left(i \mathbf{k}_{n_{x}, n_{y}} \cdot \mathbf{r}\right)
\end{aligned}
$$


where $\mathbf{k}_{n_{x}, n_{y}}=\left(2 \pi n_{x} / L_{x}, 2 \pi n_{y} / L_{y}\right)$ and $\mathbf{r}=(x, y)$. Since this method has been used extensively in the existing literature on water waves (see for example the different methods of Dommermuth \& Yue [14], Fructus et al. [19] and Christiansen et al. [5]) we are at this point content with stating that our computation of the various derivatives of $\eta$ and $\Phi_{s}$ is based on the fast Fourier transform and that we compute all nonlinear products in physical space without any dealiasing procedure.

The spatial discretization leaves a set of ordinary differential equations that describe the time evolution of the values of $\eta$ and $\Phi_{s}$ at the grid points. We integrate these equations in time using the classical fourth order RungeKutta method with a constant step size $\Delta t$. Anticipating the course of events, we have found that the time integration becomes unstable in a number of situations, and to overcome this instability we use artificial damping. We will at ter stages explicitly mention if damping is used, and when applied, it consists of multiplying the $\left(n_{x}, n_{y}\right)$ th Fourier coefficients of $\eta$ and $\Phi_{s}$ by the number

$$
D_{n_{x}, n_{y}}= \begin{cases}1 & \text { if } \kappa_{n_{x}, n_{y}} \leq \alpha \kappa_{N_{x}, N_{y}} \\ \exp \left(-36\left(\frac{\kappa_{n_{x}, n_{y}}}{\kappa_{N_{x}, N_{y}}}\right)^{12}\right) & \text { otherwise }\end{cases}
$$

- fter every time step. Here $\kappa_{n_{x}, n_{y}}=\left|\mathbf{k}_{n_{x}, n_{y}}\right|$ and $0<\alpha<1$ is a parameter to be specified. We note that this way of Jamping the time integration is a special case of the damping described in Section 5 in the textbook by Hesthaven \& varburton [20].

\section{.2 Solving the Laplace equation and computing $w_{s}$}

pur strategy to solve the Laplace equation is based on a volumetric approach, and because this can be a rather costly affair in terms of computation time, we have used an artificial boundary condition as proposed by Nicholls [12]. The idea behind this artificial boundary condition is to reduce the size of the fluid domain such that fewer degrees of freedom are needed. We here duly note that the idea has been used by several authors previously and we refer to the paper by Nicholls and the references therein. Very briefly, our method consists of the following steps: First we introduce the artificial boundary condition to limit the vertical extent of the fluid domain. Second, we map the reduced fluid comain to a time independent box using a simple stretching of the vertical coordinate. As it is non-conformal, this hange of coordinates transforms the Laplace equation, and in the third step of our method we solve this transformed Laplace equation using a pseudospectral method.

Since solving the Laplace equation does not involve time, we will suppress the time dependence of the functions $\eta$, and $w_{s}$ for notational convenience in this section.

\subsubsection{Reducing the vertical extent of the fluid domain by using an artificial boundary condition}

To introduce the artificial boundary condition we start by letting $b$ denote a positive real number such that the level $z=-b$ lies between the seabed and the lowest point of the surface elevation. As will be explained in the following,

he vertical position of the artificial boundary condition is taken as $z=-b$, and formally we choose $b$ such that $h<-b<\min \eta(x, y)$ where the minimum is taken over the rectangle $\left[0, L_{x}\right] \times\left[0, L_{y}\right]$. Dividing the fluid domain into an upper part with $-b<z<\eta(x, y)$ and a lower part with $-h<z<-b$, the Laplace problem consisting of (1a)

ind (1b) supplemented with the boundary condition $\left.\Phi\right|_{z=\eta}=\Phi_{s}$ can be restated as Laplace problems on the upper nd lower parts of the domain together with a matching condition at $z=-b$ (see Nicholls [12]). We write the Laplace roblem on the upper part of the domain as

$$
\begin{array}{ll}
\frac{\partial^{2} \Phi_{U}}{\partial x^{2}}+\frac{\partial^{2} \Phi_{U}}{\partial y^{2}}+\frac{\partial^{2} \Phi_{U}}{\partial z^{2}}=0 & \text { for }-b<z<\eta(x, y), \\
\Phi_{U}=\Phi_{s} & \text { for } z=\eta(x, y),
\end{array}
$$

the Laplace problem on the lower part of the domain as

$$
\begin{array}{ll}
\frac{\partial^{2} \Phi_{L}}{\partial x^{2}}+\frac{\partial^{2} \Phi_{L}}{\partial y^{2}}+\frac{\partial^{2} \Phi_{L}}{\partial z^{2}}=0 & \text { for }-h<z<-b, \\
\frac{\partial \Phi_{L}}{\partial z}=0 & \text { for } z=-h,
\end{array}
$$

and the matching condition at $z=-b$ as

$$
\begin{array}{ll}
\Phi_{U}=\Phi_{L} & \text { for } z=-b, \\
\frac{\partial \Phi_{U}}{\partial z}=\frac{\partial \Phi_{L}}{\partial z} & \text { for } z=-b .
\end{array}
$$


The point of doing this domain decomposition is that we can solve (6) analytically, if we for the moment assume the function $\phi=\left.\Phi_{U}\right|_{z=-b}$ to be known. In the case where the horizontal part of $\Phi_{U}$ is approximated by a truncated Fourier series with $2 N_{x} \times 2 N_{y}$ coefficients, we may write

$$
\phi(x, y)=\sum_{n_{x}=-N_{x}}^{N x-1} \sum_{n_{y}=-N_{y}}^{N_{y}-1} \widehat{\phi}_{n_{x}, n_{y}} \exp \left(i \mathbf{k}_{n_{x}, n_{y}} \cdot \mathbf{r}\right)
$$

and it immediately follows that the unique solution of (6) is given by

$$
\Phi_{L}(x, y, z)=\sum_{n_{x}=-N_{x}}^{N_{x}-1} \sum_{n_{y}=-N_{y}}^{N_{y}-1} \widehat{\phi}_{n_{x}, n_{y}} \exp \left(i \mathbf{k}_{n_{x}, n_{y}} \cdot \mathbf{r}\right) \frac{\cosh \left(\kappa_{n_{x}, n_{y}}(h+z)\right)}{\cosh \left(\kappa_{n_{x}, n_{y}}(h-b)\right)}
$$

which in turn implies that

$$
\frac{\partial \Phi_{L}}{\partial z}(x, y,-b)=\sum_{n_{x}=-N_{x}}^{N_{x}-1} \sum_{n_{y}=-N_{y}}^{N_{y}-1} \widehat{\phi}_{n_{x}, n_{y}} \kappa_{n_{x}, n_{y}} \tanh \left(\kappa_{n_{x}, n_{y}}(h-b)\right) \exp \left(i \mathbf{k}_{n_{x}, n_{y}} \cdot \mathbf{r}\right) .
$$

we now define the operator $T$ by the action

$$
T[\phi]=\sum_{n_{x}=-N_{x}}^{N_{x}-1} \sum_{n_{y}=-N_{y}}^{N_{y}-1} \widehat{\phi}_{n_{x}, n_{y}} \kappa_{n_{x}, n_{y}} \tanh \left(\kappa_{n_{x}, n_{y}}(h-b)\right) \exp \left(i \mathbf{k}_{n_{x}, n_{y}} \cdot \mathbf{r}\right)
$$

nd combine this with the matching condition $(7 \mathrm{~b})$, we can write the Laplace problem on the upper part of the domain as

$$
\begin{array}{ll}
\frac{\partial^{2} \Phi_{U}}{\partial x^{2}}+\frac{\partial^{2} \Phi_{U}}{\partial y^{2}}+\frac{\partial^{2} \Phi_{U}}{\partial z^{2}}=0 & \text { for }-b<z<\eta(x, y), \\
\Phi_{U}=\Phi_{s} & \text { for } z=\eta(x, y), \\
\frac{\partial \Phi_{U}}{\partial z}(x, y,-b)-T\left[\Phi_{U}(x, y,-b)\right]=0 &
\end{array}
$$

which is a problem for $\Phi_{U}$ whose vertical range is not the original interval $[-h, \eta(x, y)]$, but rather the interval $-b, \eta(x, y)$ ]. Since $b$ in many cases might be chosen much smaller than $h$ - in particular on deep water - this offers us the possibility of using fewer collocation points in the vertical dimension than if we had attempted to solve the original roblem (1a) directly. We also note, however, that the operator $T$ depends on $b$, meaning that if $b$ is changed during a simulation, then the matrix representing the action of $T$ must be recalculated.

\section{Mapping the reduced fluid domain to a time independent rectangular box}

Tn order to solve the Laplace problem (12) we map the reduced fluid domain to a time independent rectangular box hrough the coordinate transformation $(x, y, z) \mapsto(x, y, s)$ where $s$ is given by

$$
s(x, y, z)=\frac{2 z+b-\eta(x, y)}{b+\eta(x, y)} \text {. }
$$

As $z$ varies between $-b$ and $\eta(x, y)$ it follows that $s$ lies in the interval $[-1,1]$. If we now define the function $F$ by
$(x, y, s(x, y, z))=\Phi_{U}(x, y, z)$ it follows from (12a), the chain rule and the definition of $s$ that $F$ must satisfy the equation

$$
0=\frac{\partial^{2} F}{\partial x^{2}}+\frac{\partial^{2} F}{\partial y^{2}}+\left(\left(\frac{\partial s}{\partial x}\right)^{2}+\left(\frac{\partial s}{\partial y}\right)^{2}+\left(\frac{\partial s}{\partial z}\right)^{2}\right) \frac{\partial^{2} F}{\partial s^{2}}+2 \frac{\partial s}{\partial x} \frac{\partial^{2} F}{\partial x \partial s}+2 \frac{\partial s}{\partial y} \frac{\partial^{2} F}{\partial y \partial s}+\left(\frac{\partial^{2} s}{\partial x^{2}}+\frac{\partial^{2} s}{\partial y^{2}}\right) \frac{\partial F}{\partial s}
$$

and after inserting the derivatives of $s$ and multiplying both sides of the equation by $(b+\eta)^{2}$ it becomes

$$
\begin{aligned}
0= & (b+\eta)^{2}\left(\frac{\partial^{2} F}{\partial x^{2}}+\frac{\partial^{2} F}{\partial y^{2}}\right)+\left(4+(1+s)^{2}\left(\left(\frac{\partial \eta}{\partial x}\right)^{2}+\left(\frac{\partial \eta}{\partial y}\right)^{2}\right)\right) \frac{\partial^{2} F}{\partial s^{2}} \\
& -2(b+\eta)(1+s)\left(\frac{\partial \eta}{\partial x} \frac{\partial^{2} F}{\partial x \partial s}+\frac{\partial \eta}{\partial y} \frac{\partial^{2} F}{\partial y \partial s}\right) \\
& +(1+s)\left(2\left(\left(\frac{\partial \eta}{\partial x}\right)^{2}+\left(\frac{\partial \eta}{\partial y}\right)^{2}\right)-(b+\eta)\left(\frac{\partial^{2} \eta}{\partial x^{2}}+\frac{\partial^{2} \eta}{\partial y^{2}}\right)\right) \frac{\partial F}{\partial s}
\end{aligned}
$$


In addition to this equation, $F$ must satisfy boundary conditions at $s=1$ and $s=-1$. The boundary condition on $F$ at $s=1$ can be deduced from (12b) and is simply

$$
\left.F\right|_{s=1}=\Phi_{s}
$$

while the boundary condition on $F$ at $s=-1$ follows from (12c) and reads

$$
\frac{2}{b+\eta} \frac{\partial F}{\partial s}(x, y,-1)-T[F(x, y,-1)]=0 .
$$

\subsection{Discretization and solution of the transformed Laplace equation}

Tho solve the problem consisting of (15), (16) and (17) we employ a pseudospectral method and seek to determine the values of $F$ on the grid

$$
\left\{\left(x_{n_{x}}, y_{n_{y}}, s_{n_{s}}\right) \mid 0 \leq n_{x}<2 N_{x}, \quad 0 \leq n_{y}<2 N_{y} \text { and } 0 \leq n_{s}<N_{s}+1\right\}
$$

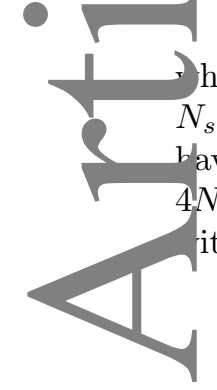

here $x_{n_{x}}$ and $y_{n_{y}}$ are given by (2) and $s_{n_{s}}$ is the $n_{s}$ th point of the Legendre-Gauss-Lobatto quadrature of order $N_{s}$ ordered such that $s_{0}=-1$. We require the boundary condition (16) to be satisfied at the set of points that blave $n_{s}=N_{s}$ and the boundary condition (17) to be satisfied at the set of points with $n_{s}=0$. At the remaining $4 N_{x} N_{y}\left(N_{s}-1\right)$ points we require the differential equation (15) to be satisfied. We compute all nonlinear products ithout any dealiasing procedure, and to compute the derivatives of $F$ on the grid, we assume $F$ to be of the form

$$
F(x, y, s)=\sum_{n_{x}=-N_{x}}^{N_{x}-1} \sum_{n_{y}=-N_{y}}^{N_{y}-1} \sum_{n_{s}=0}^{N_{s}} \widehat{F}_{n_{x}, n_{y}, n_{s}} \exp \left(i \mathbf{k}_{n_{x}, n_{y}} \cdot \mathbf{r}\right) l_{n_{s}}(s)
$$

$$
l_{n_{s}}(s)=\prod_{\substack{p=0 \\ p \neq n_{s}}}^{N_{s}} \frac{s-s_{p}}{s_{n_{s}}-s_{p}}
$$

the $n_{s}$ th Lagrange polynomial of order $N_{s}$. With $F$ of this form, its derivatives in the $x$ - and $y$-dimensions can be computed using fft-based differentiation, while its derivatives in the $s$-dimension require the derivatives of $l_{n_{s}}$ which on be computed using the procedure outlined in Section 3.5.2 in the book by Kopriva [21].

The above method of satisfying the boundary conditions and the differential equation at the set of points (18) generates a system of $4 N_{x} N_{y}\left(N_{s}+1\right)$ linear equations for the values of $F$ on the grid (18), and we will denote these quations collectively by $\mathcal{A} \mathbf{x}=\mathbf{b}$ where $\mathcal{A}$ is a matrix and $\mathbf{x}$ and $\mathbf{b}$ are column vectors. This system may be solved I a number of different ways and we have chosen to use the iterative method GMRES (see Saad \& Schultz [22]) with relative tolerance $\epsilon$, mainly because it does not need the matrix $\mathcal{A}$ to be explicitly constructed but only a means of mputing the product of $\mathcal{A}$ with a vector. Despite this advantage, the computational cost of GMRES can still be prohibitive if no preconditioner is applied to the problem. In order to construct a preconditioner, we follow the idea of Fuhrman \& Bingham [23] and consider the problem consisting of (15), (16) and (17) with $\eta=0$, i.e.

$$
\begin{aligned}
& 0=b^{2}\left(\frac{\partial^{2} F}{\partial x^{2}}+\frac{\partial^{2} F}{\partial y^{2}}\right)+4 \frac{\partial^{2} F}{\partial s^{2}} \\
& F(x, y, 1)=\Phi_{s}(x, y) \\
& \frac{2}{b} \frac{\partial F}{\partial s}(x, y,-1)-T[F(x, y,-1)]=0 .
\end{aligned}
$$

In terms of efficiency, this problem is attractive as a preconditioning problem because it is easily solved for the expansion coefficients of $F$, and because it is time independent, meaning that it suffices to factor it once. Regarding the resemblance of the original problem, we note that (21) is obtained from the original problem in the limit of negligible wave steepness and therefore should provide a good approximation. In fact, we have found the iteration count to be around 10 or less even for the most nonlinear cases, when combining the preconditioning strategy with using an appropriate linear combination of solutions to the Laplace problem at the three previous time steps as the initial guess. 
horizontal velocity, $u=\partial_{x} \Phi$, below the wave crest, we also investigate the stability and accuracy of the time integration with and without artificial damping. Despite the fact that steady nonlinear waves are essentially two-dimensional, they provide an interesting test case because their time evolution is known (numerically) exactly. In addition, they represent a great computational challenge as they may contain a sustained degree of nonlinearity hardly found anywhere else. Taking the wavelength, $\lambda$, to be fixed, we characterize the waves by their relative water depth $k h$ and their relative height $H / H_{\max }$, where $k=2 \pi / \lambda$ is the fundamental wave number, $H$ is the vertical distance from the trough to the crest and $H_{\max }$ is the ( $h$ and $\lambda$ dependent) maximal wave height corresponding to wave breaking. According to Battjes [24], the maximum steepness is given by

$$
\text { (1) } \frac{H_{\max }}{\lambda}=0.1401 \tanh (0.8863 k h)
$$

hich is the formula we use to calculate the values of $H_{\max }$. For completeness, values for the wave steepness $H / \lambda$ will also be reported. Throughout this section we will only consider a single wavelength and hence take $L_{x}=\lambda$. The value $L_{y}$ can be chosen arbitrarily and in this work we take $L_{y}=\lambda$. Moreover, we take $N_{y}=1$ and initialize $\eta$ and $\Phi_{s}$ such that $\eta\left(x, y_{0}\right)=\eta\left(x, y_{1}\right)$ and $\Phi_{s}\left(x, y_{0}\right)=\Phi_{s}\left(x, y_{1}\right)$ for all values of $x$. Finally, if not explicitly stated, we choose the location of the artificial boundary condition by setting $b=\max \eta(x, y)$ where the maximum is taken over all grid points. We note that this choice makes sense, because for steady nonlinear waves the crest is further away from the ero level than the trough.

Regarding the initialization of the surface quantities, there is a rich literature of accurate methods for computing eady nonlinear waves, among which the method of Rienecker \& Fenton [25] is probably the most widely used. Here ye have chosen to use the method of Clamond \& Dutykh [26] for initializing $\eta$ and $\Phi_{s}$ as well as for providing the a arget solution for the vertical surface velocity, $w_{s}^{T}$. When executed in double precision, this method is capable of computing the surface quantities of all but the highest waves correct to double precision in a very small amount of time, but it gives the values of the surface quantities on a grid which is not equidistant. For that reason we use the method of Clamond \& Dutykh to produce the values of $\eta, \Phi_{s}$ and $w_{s}^{T}$ at a very large number of non-equidistant points (typically around 100,000), and using interpolation we compute the values of $\eta$ and $\Phi_{s}$ on an equidistant grid with $\Omega N_{x}$ points while we compute the values of $w_{s}^{T}$ on an equidistant grid with $2 N_{T}+1$ points. Here $N_{T}$ is the smallest i teger such that $w_{s}^{T}$ is accurate to double precision, by which we mean that $\widehat{w}_{1}^{T} / \widehat{w}_{N_{T}}^{T}=O\left(10^{-14}\right)$ where $\widehat{w}_{n}^{T}$ is the th Fourier coefficient of $w_{s}^{T}$. In all cases we will have $N_{T}>N_{x}$.

When computing $w_{s}$ from $\eta$ and $\Phi_{s}$ with our method in the sections below, we assess the accuracy of the computation $y$ measuring the error of $w_{s}$ relative to the target velocity, $w_{s}^{T}$. Having computed $w_{s}$ we measure its error using the $L^{2}$-norm, i.e.

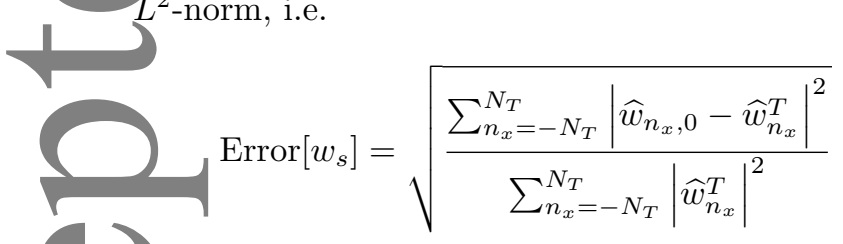

here it is understood that $w_{s}$ has been zero-padded such that it contains $2 N_{T}+1$ coefficients.

\section{.1 Accuracy of $w_{s}$ as a function of $N_{x}$}

To begin with, we assess our numerical method by testing the convergence of the computed $w_{s}$ to the numerically xact result as a function of $N_{x}$ for fixed values of $N_{s}$ and with $\epsilon=10^{-14}$. We perform the test in shallow water with $\kappa h=0.3$, at an intermediate water depth with $k h=1$ and on deep water with $k h=2 \pi$, and for each value of $k h$ we onsider $H / H_{\max }=0.1,0.5,0.9$ and 0.99 . The values of $N_{T}$ and $H / \lambda$ for each of the cases are listed in tables 1 and 2 , respectively, and we note that with the choice of $b$, the domain size is reduced roughly by the factor $1-b / h$ which is about $90 \%$ or more in the deep water case, about $55 \%$ or more in the intermediate case and about $33 \%$ or more in the shallow water case.

The results of the computation are shown in Figure 2 and from these we conclude that if $N_{x}$ and $N_{s}$ are chosen large enough, our numerical method is capable of computing $w_{s}$ to about 12 significant digits in a stable way for all the chosen values of $k h$ and $H / H_{\max }$. We emphasize that this is a property not shared by many numerical methods for water wave. For example Dommermuth \& Yue [14] showed that with their HOS method, which we note is widely used today, the computation of $w_{s}$ for steady nonlinear waves in deep water becomes unstable when the steepness reaches roughly $H / H_{\max } \approx 0.8$. In this connection it should be noted that computing $w_{s}$ correct to 12 digits for a steady wave in deep water with $H / H_{\max }=0.8$ is a much easier task than for a wave with $H / H_{\max }=0.99$, since much larger $N_{x}$ and $N_{s}$ must be used in the latter case. 

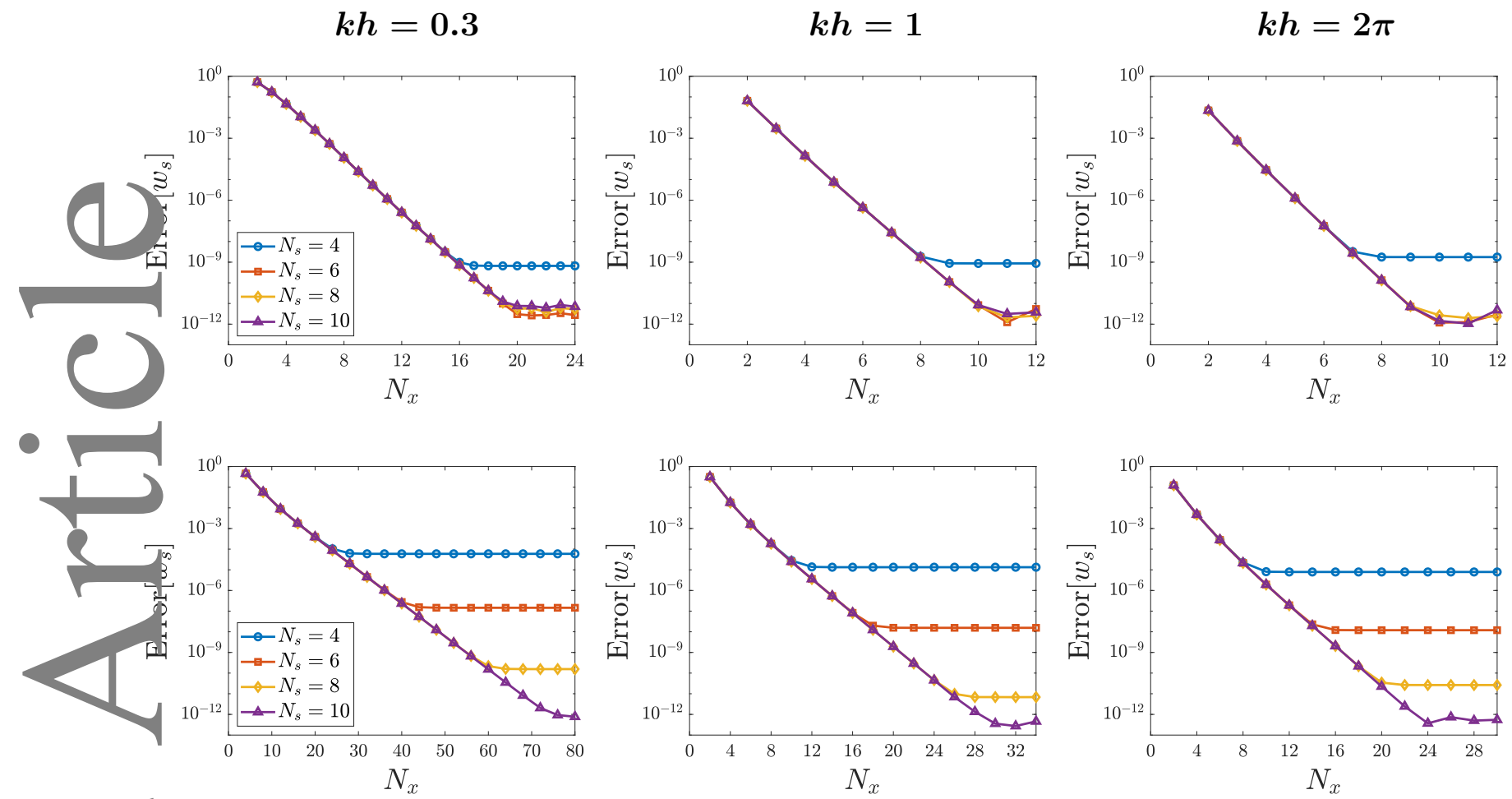

0.10
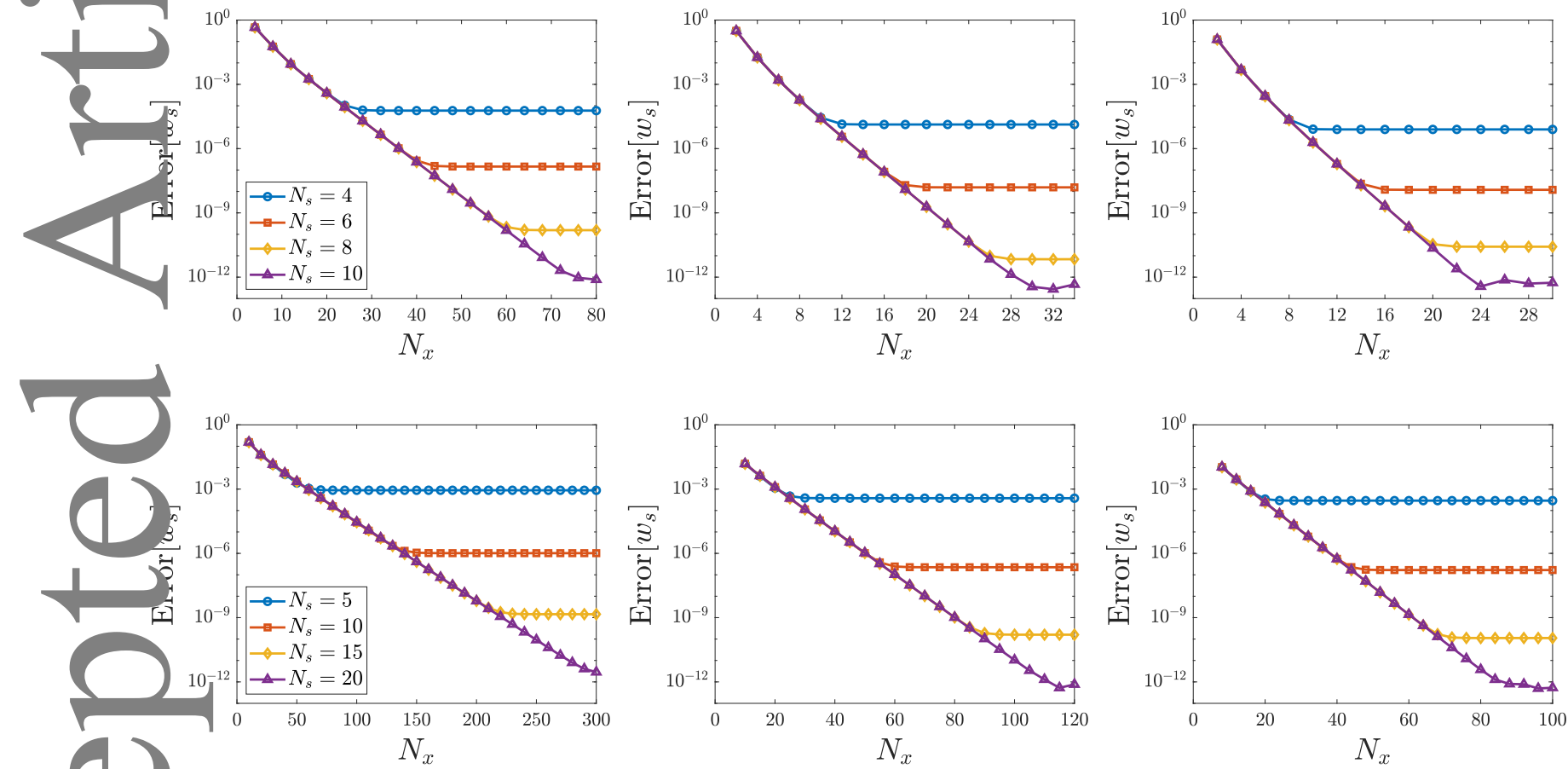

0.50
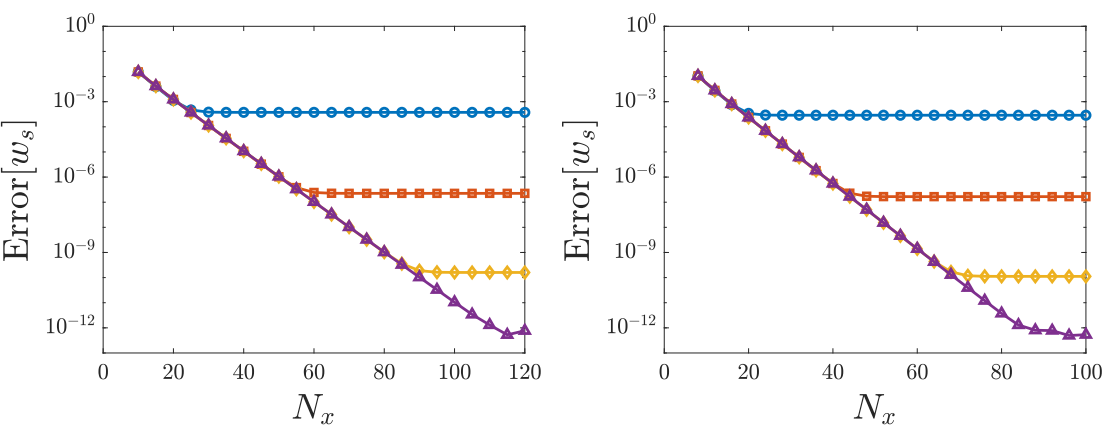

0.90
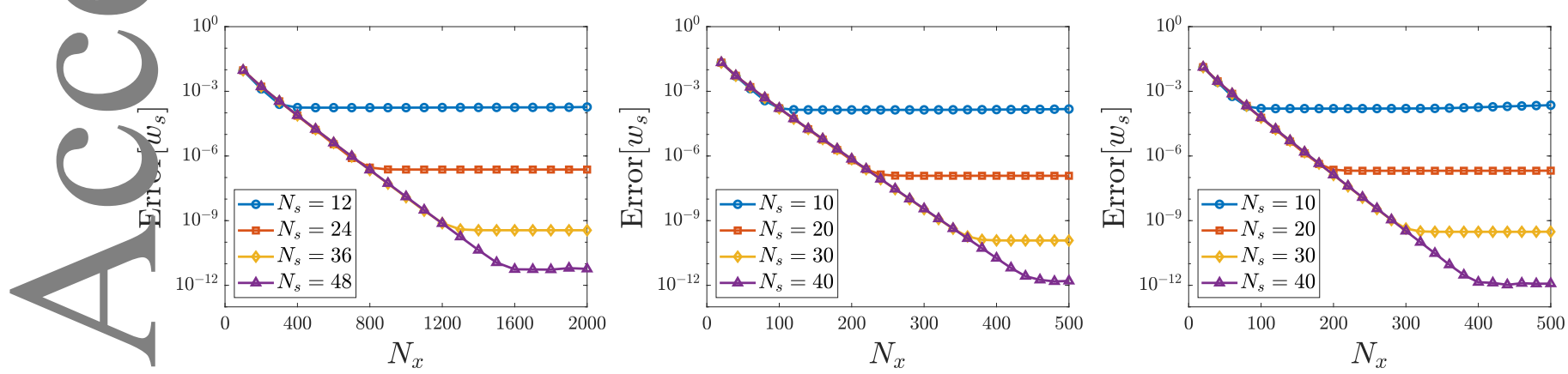

0.99

Figure 2: The convergence of $w_{s}$ to the exact result as a function of $N_{x}$ for different values of $N_{s}$. Starting from the top, the rows correspond to $H / H_{\max }=0.1,0.5,0.9$ and 0.99 , respectively, while the columns from left to right correspond to the shallow, intermediate and deep water cases, respectively. For the figures with no legend, the legend of the figure in the same row and first column applies. 


\begin{tabular}{lcccc}
\hline & \multicolumn{4}{c}{$H / H_{\max }$} \\
\cline { 2 - 5 }$k h$ & 0.1 & 0.5 & 0.9 & 0.99 \\
\hline 0.3 & 25 & 80 & 310 & 2000 \\
1 & 15 & 35 & 120 & 500 \\
$2 \pi$ & 12 & 30 & 100 & 500 \\
\hline
\end{tabular}

Table 1: The values of $N_{T}$ used for the convergence study of $w_{s}$ as a function of the relative water depth and the relative wave height.

\begin{tabular}{lccccc}
\hline & \multicolumn{5}{c}{$H / H_{\max }$} \\
\cline { 2 - 6 }$k h$ & 0.1 & 0.5 & 0.9 & 0.99 & 1 \\
\hline 0.3 & 0.0036 & 0.0182 & 0.0327 & 0.0360 & 0.0364 \\
1 & 0.0099 & 0.0470 & 0.0894 & 0.0984 & 0.0994 \\
$2 \pi$ & 0.0140 & 0.0700 & 0.1260 & 0.1387 & 0.1401 \\
\hline
\end{tabular}

able 2: The values of $H / \lambda$ as a function of the relative water depth and the relative wave height.

\section{.2 Smallest $N_{x}$ and $N_{s}$ to achieve $w_{s}$ to a certain accuracy}

1 the previous section we provided evidence that our numerical method can compute $w_{s}$ correct up to 12 significant digits for steady nonlinear waves at virtually all values of $H / H_{\max }$ and $k h$, provided that we are willing to use fine enough resolution. For most engineering problems 12 digits of accuracy are, however, much more than what is actually needed, and hence a significant amount of time may be saved by using just the right amount of collocation points to meet the accuracy requirements. For that reason we devote this section to finding the smallest values of $N_{x}$ and $N_{s}$ uch that Error $\left[w_{s}\right]<\tau$ where $\tau$ is some small number. We will denote these smallest values of $N_{x}$ and $N_{s}$ by $N_{x}^{*}$ and $Y_{s}^{*}$, respectively, and compute them for the cases where $\tau=10^{-4}, 10^{-5}$ and $10^{-6}$. In order to make sure that the rror on $w_{s}$ is not affected by the GMRES method, we take $\epsilon=10^{-14}$.

Now, suppose that we are given $\tau$ and want to find $N_{x}^{*}$ and $N_{s}^{*}$. From the convergence results given in figure 2 we onclude that $N_{s}=24$ is large enough that $w_{s}$ can be computed with a relative error less than $10^{-6}$ in all cases, and our algorithm for finding $N_{x}^{*}$ and $N_{s}^{*}$ is therefore the following:

1. Let $H / H_{\max }$ and $k h$ be given and set $N_{s}=24$. Moreover, set $N_{x}=2$.

2. Compute Error $\left[w_{s}\right]$ for these values of $N_{x}$ and $N_{s}$. If Error $\left[w_{s}\right] \leq \tau$ then $N_{x}^{*}=N_{x}$ and we continue to the next step. If on the other hand Error $\left[w_{s}\right]>\tau$, we increase $N_{x}$ by 1 and repeat this step with the new value of $N_{x}$.

3. Having found $N_{x}^{*}$ we set $N_{s}=2$ and compute Error $\left[w_{s}\right]$ using this value of $N_{s}$ together with $N_{x}^{*}$. If Error $\left[w_{s}\right] \leq \tau$ we have $N_{s}^{*}=N_{s}$. If, however, Error $\left[w_{s}\right]>\tau$ we increase $N_{s}$ by 1 and repeat this step until we have found the smallest value of $N_{s}$.

Ne have executed this algorithm for $k h=0.3,1$ and $2 \pi$ with $0.01 \leq H / H_{\max } \leq 0.99$, and the obtained values of $N_{x}^{*}$ and $N_{s}^{*}$ are shown as functions of the wave height in figure 3 and figure 4 for the three different values of $\tau$. loreover, the values of $N_{x}^{*}$ and $N_{s}^{*}$ when computed with $\tau=10^{-5}$ and $\tau=10^{-6}$ relative to the values of $N_{x}^{*}$ and $N_{s}^{*}$ when computed with $\tau=10^{-4}$ are shown in the figures. We conclude that if one is only interested in computing $s$ with a relative error less than $10^{-4}$, then in many situations, and almost regardless of $k h$, the required horizontal resolution can be reduced by about $25 \%$ or more compared to when computing $w_{s}$ with a relative error of $10^{-5}$, and by about $40 \%$ or more compared to when computing $w_{s}$ with a relative error of $10^{-6}$. In addition we conclude that the "equired vertical resolution can be reduced by roughly the same amount. Besides the possible computational savings it is interesting to note that the value of $N_{x}^{*}$ grows exponentially as $H / H_{\max }$ approaches 1 . In practice this means that a Fourier series description of regular waves is not of much use for the very steepest waves simply because too many degrees of freedom are needed to obtain accurate results.

Besides comparing the present method to itself, it is of course also interesting to compare it to other methods in order to see the resolution needed to achieve $w_{s}$ with a certain accuracy. For that reason we have computed $N_{x}^{*}$ and $N_{s}^{*}$ using our recent Galerkin method (see Klahn et al. [10]) which discretizes the entire fluid domain instead of using an artificial boundary condition as done here but otherwise may be though of as an alias-free two-dimensional version of the present method. We have found that, despite the fact that the Galerkin method is free of aliasing errors, the required horizontal resolution is almost exactly the same as for the present method for all water depths. 

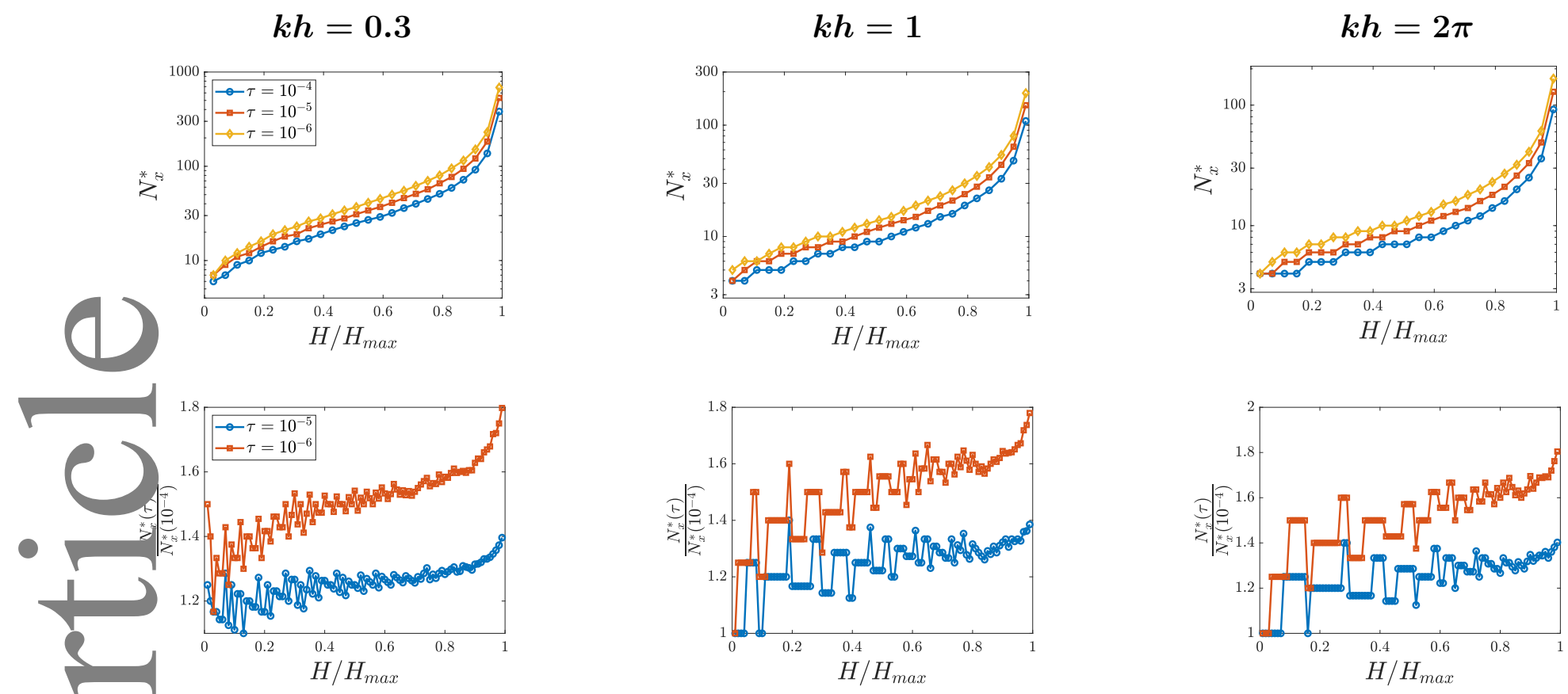

igure 3: First row: $N_{x}^{*}$ as a function of the relative wave height for different values of the relative water depth $k h$ and the tolerance $\tau$. Second row: The value of $N_{x}^{*}$ with tolerance $\tau$ relative to $N_{x}^{*}$ with tolerance $10^{-4}$ as a function of the relative wave height for different values of the relative water depth $k h$. The legends apply rowwise.

Regarding the required vertical resolution, we have found that for $k h=0.3$ and $k h=1$ the two methods again require Imost the same resolution. For $k h=2 \pi$ our results show that the present method only requires about half the vertical solution compared to the Galerkin method, and we therefore conclude that substantial savings may be obtained using the artificial boundary condition for deep water problems, while practically nothing is gained by using the boundary (1) ondition for shallow to intermediately deep water problems.

\subsection{The accuracy of $w_{s}$ as a function of $\epsilon$}

So far we have taken $\epsilon=10^{-14}$ in all our calculations in order to test the behavior of Error $\left[w_{s}\right]$ when the spatial resolution is varied. Having found $N_{x}^{*}$ and $N_{s}^{*}$ needed to compute $w_{s}$ such that Error $\left[w_{s}\right]<\tau$, an obvious question lether we can use a larger (i.e. less restrictive) value of $\epsilon$ without losing accuracy and if so, if we can reduce the number of GMRES iterations by using the larger value of $\epsilon$.

To find out, we have computed the error of $w_{s}$ and monitored the number of GMRES iterations as a function of $\epsilon$ for different values of $\tau, k h$ and $H / H_{\max }$ when using $N_{x}=N_{x}^{*}$ and $N_{s}=N_{s}^{*}$. figure 5 shows our results for the

ase where $k h=0.3$ and $H / H_{\max }=0.95$ (i.e. $H / \lambda=0.0346$ ), and from the figure it is clear that Error $\left[w_{s}\right]$ stagnates ong before $\epsilon=10^{-14}$, meaning that a larger value of $\epsilon$ can be used while still achieving Error $\left[w_{s}\right]<\tau$. Moreover, the figure illustrates that the number of iterations keeps increasing when $\epsilon$ decreases, even though the error of $w_{s}$ becomes onstant. Combined, these two results thus imply that the computation of $w_{s}$ may be executed significantly faster without any loss of accuracy simply by choosing a larger value for $\epsilon$.

The results in figure 5 of course only constitute a single example and are computed without utilizing earlier solutions the Laplace problem to form an initial guess. We have, however, found the results for this case to be representative for many others, including small, intermediate and large values of $k h$ and $H / H_{\max }$ as well as during time integration where an initial guess is used. Given $\tau$, choosing $\epsilon=\tau / 10^{3}$ seems in general to be a small enough tolerance to avoid errors from the GMRES method, and for relatively small heights $\epsilon$ can typically be chosen significantly larger, for example $\epsilon=\tau / 50$.

\subsection{Computation of velocity profiles}

The calculation of the horizontal velocity, $u=\partial_{x} \Phi$, as a function of $z$ below the wave crest of steep regular waves is a demanding problem that has only been attempted by a very limited number of authors. We mention here Madsen et al. [27], who used a Boussinesq-type model, and Engsig-Karup et al. [9], who used a nodal spectral element method. Madsen et al. did their test with $k h=10$ and $H / H_{\max }=0.96$ (i.e. $H / \lambda=0.135$ ) and found that their result was 

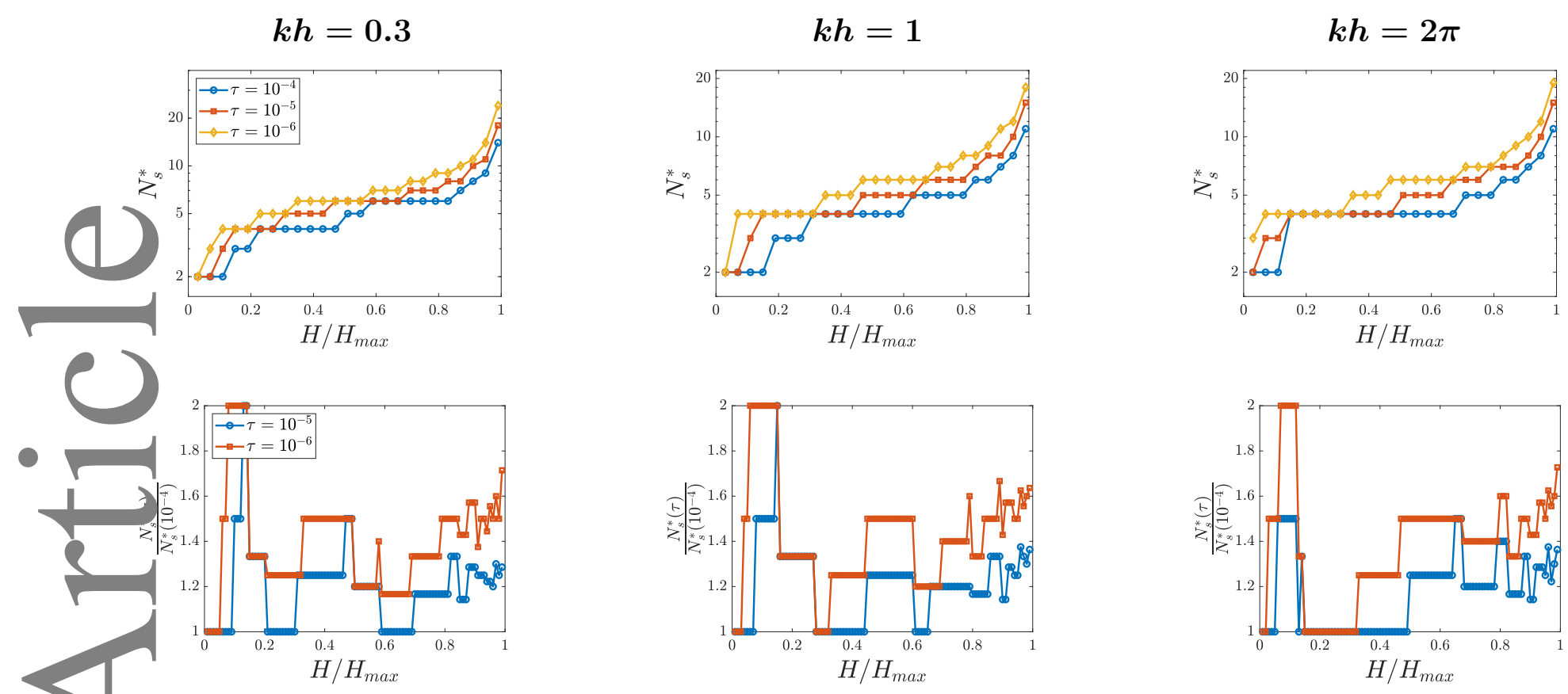

igure 4: First row: $N_{s}^{*}$ as a function of the wave steepness relative to the maximum steepness (24) for different values of the relative water depth $k h$ and the tolerance $\tau$. Second row: The value of $N_{s}^{*}$ with tolerance $\tau$ relative to $N_{s}^{*}$ with folerance $10^{-4}$ as a function of the wave steepness relative to the maximum steepness (24) for different values of the lative water depth $k h$. The legends apply row wise.
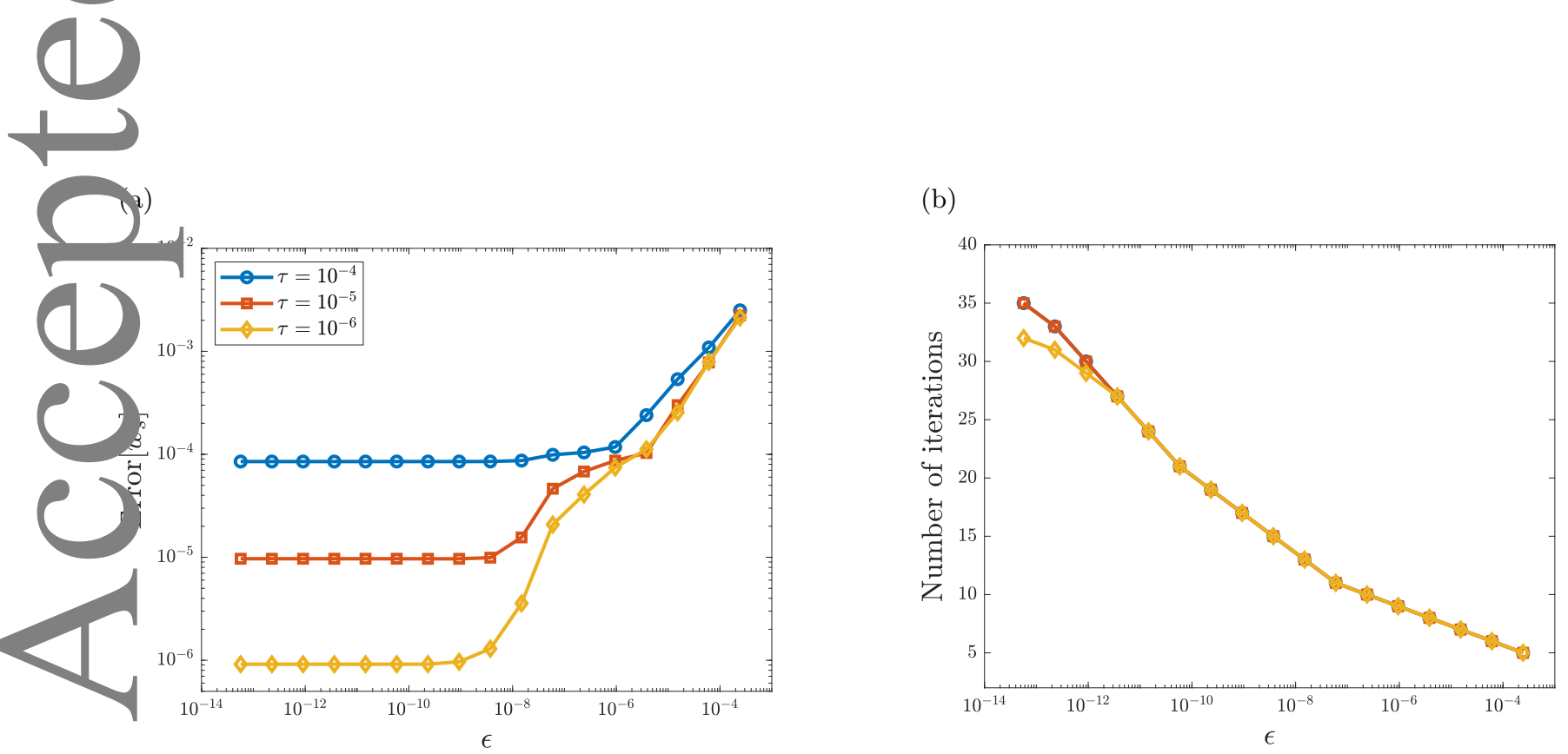

Figure 5: (a): The error of $w_{s}$ as a function of the relative tolerance, $\epsilon$, used in GMRES for different values of $\tau$ with $k h=0.3$ and $H / H_{\max }=0.95$ corresponding to $H / \lambda=0.0346$. (b): The number of GMRES iterations as a function of the relative tolerance $\epsilon$ for different values of $\tau$ and the same water depth and wave height as in (a). The legend applies to both figures. 


\begin{tabular}{lcc}
\hline & \multicolumn{2}{c}{$H / H_{\max }$} \\
\cline { 2 - 3 }$k h$ & 0.95 & 0.99 \\
\hline 0.3 & $7.5 \times 10^{-6}$ & $1.8 \times 10^{-3}$ \\
1 & $1.5 \times 10^{-5}$ & $3.0 \times 10^{-4}$ \\
10 & $6.4 \times 10^{-6}$ & $6.9 \times 10^{-4}$ \\
\hline
\end{tabular}

Table 3: The relative difference between the results for $u / c$ at the crest of our method and the stream function method for different values of $k h$ and $H / H_{\max }$. The results have been obtained using the parameters $N_{x}=400, N_{s}=100$, $=10^{-14}$ and $b=\max |\eta(x, y)|$ for $k h=0.3$ and $k h=1$ and $b=10 \times \max |\eta(x, y)|$ for $k h=10$.

1h good agreement with the result obtained from the stream function method of Rienecker \& Fenton [25] close to the
rest while their result was visibly inaccurate close to the seabed. Engsig-Karup et al. considered the cases $k h=1$
hd $k h=10$ with $H / H_{\max }=0.95$ (corresponding to $H / \lambda=0.10$ and $H / \lambda=0.133$, respectively). For both cases they
found their method to be most accurate around the seabed and least accurate around the wave crest. Reading off
peir figure 8 , it may be seen that the error (when compared to the result obtainable by the method of Rienecker \&
Fenton) of $u / c$ at the wave crest, with $c$ the wave celerity, was roughly $4 \times 10^{-3}$ for the intermediate water depth and
$15 \times 10^{-3}$ for the deep water case.

In this work we consider the computation of $u(z)$ below the wave crest of waves with $H / H_{\max }=0.95$ and $H / H_{\max }=$ .99 for the three relative water depths $k h=0.3, k h=1$ and $k h=10$ to test how well our method can calculate the orizontal velocity for steep waves. In the upper part of the domain, i.e. $-b<z<\eta$, we have calculated $\partial_{x} \Phi$ from the solution to the transformed Laplace equation (15), $F$, as

$$
\frac{\partial \Phi}{\partial x}=\frac{\partial F}{\partial x}-\frac{1}{b+\eta}(1+s) \frac{\partial \eta}{\partial x} \frac{\partial F}{\partial s}
$$

While in the lower part of the domain, $-h<z<-b$, we have used the analytical solution (9) to calculate $\partial_{x} \Phi$ as

$$
\frac{\partial \Phi}{\partial x}=\sum_{n_{x}=-N_{x}}^{N x-1} \sum_{n_{y}=-N_{y}}^{N_{y}-1} i \frac{2 \pi n_{x}}{L_{x}} \widehat{\phi}_{n_{x}, n_{y}} \exp \left(i \mathbf{k}_{n_{x}, n_{y}} \cdot \mathbf{r}\right) \frac{\cosh \left(\kappa_{n_{x}, n_{y}}(h+z)\right)}{\cosh \left(\kappa_{n_{x}, n_{y}}(h-b)\right)} .
$$

Ve recall that $\widehat{\phi}_{n_{x}, n_{y}}$ denotes the $\left(n_{x}, n_{y}\right)$ th Fourier coefficient of the function $\phi=\left.F\right|_{s=-1}$, where $s=-1$ corresponds to $z=-b$. In all cases we have used the parameters $N_{x}=400, N_{s}=100$ and $\epsilon=10^{-14}$ and while we have taken $0=\max |\eta(x, y)|$ for the shallow and intermediately deep water cases, we have taken $b=10 \times \max |\eta(x, y)|$ for the lond water case for the simple reason that the cosh-functions in (27) easily overflow for large arguments when working in double precision. Our results for $u(z)$ below the wave crest are shown in figure 6 together with the best results we puld obtain using the stream function method of Rienecker \& Fenton. The figure shows a close match between the wo methods for all values of $k h$ and $H / H_{\max }$, and we note that besides our own recent work (see Klahn et al. [10]), seemingly nobody has attempted or been able to compute the profiles for the waves with $H / H_{\max }=0.99$. The relative ifference between the results for $u / c$ at the crest of our method and the stream function method is shown in table 3 and from these we conclude that the difference between our method and the stream function method is smaller than he difference between the method of Engsig-Karup et al. and the stream function method by about a factor 1000 hen $k h=1$ and about a factor 625 when $k h=10$.

\section{$.5 \quad$ Stability of the time integration of $\eta$ and $\Phi_{s}$ without artificial damping}

The stability of the time integration of the present method inevitably varies from case to case. The general rule seems op be that the more nonlinear the wave is and the finer it is resolved, the earlier the simulation breaks down. To illustrate this general rule, we here test the method's ability to integrate a steady nonlinear wave train in time for 50 periods without blowing up for different values of $H / H_{\max }$ and $k h$ as well as varying resolutions. We define the blowup time, $t_{b}$, as the smallest $t$ for which

$$
\max \{\eta(x, y, t)\}>2 \max \{\eta(x, y, 0)\}
$$

where the maximum is taken over all grid points. We choose $N_{x}$ and $N_{s}$ such that we initially have Error $\left[w_{s}\right]<\tau$ and take the time step to be $\Delta t=T /\left(4 N_{x}\right)$, where $T$ is the temporal period of the wave, such that the wave moves half the distance between two adjacent grid points per time step corresponding to the Courant number $C=c \Delta t / \Delta x=0.5$ 

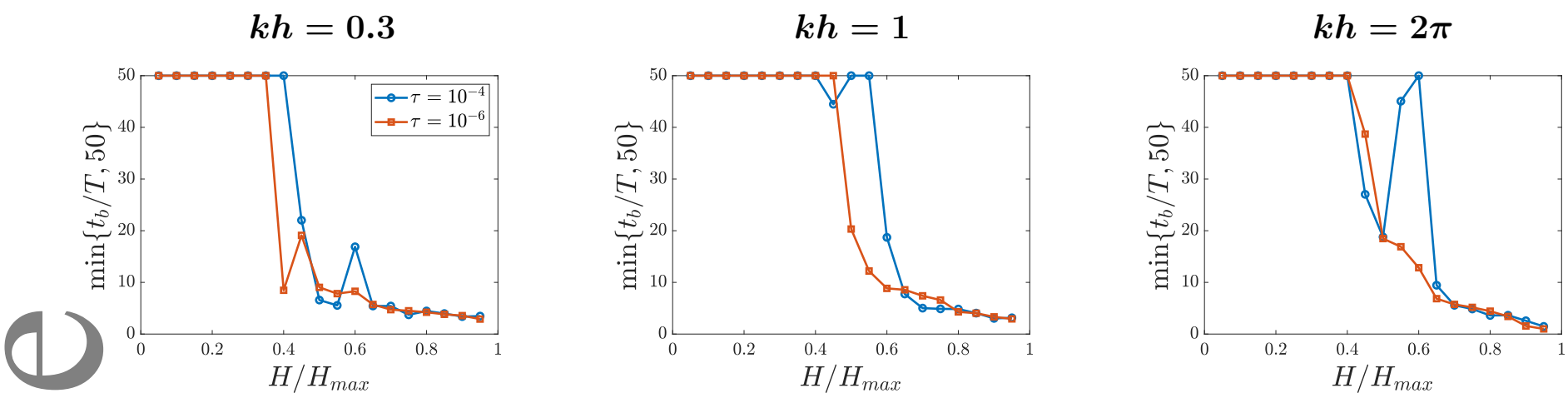

rigure 7: The quantity $\min \left\{t_{B} / T, 50\right\}$ as a function of $H / H_{\max }$ for different values of $k h$ and $\tau$. The legend applies to all figures.

ith the sophisticated numerical methods of today, accurate time integration of extremely nonlinear waves remains
gut of reach, thus motivating the search for stable algorithms. When taking $k h=1$ we have obtained similar results
sing the same parameters, but in shallow water with $k h=0.3$ we have had significantly more trouble integrating
steep waves accurately in time even when using damping. Our experiments with different parameter choices show that
r $k h=0.3$ we could get a match similar to the one in figure 8 (a) for wave heights up to about $H / H_{\text {max }}=0.8$ and
tp obtain this, we had to use less damping $(\alpha=0.9)$ and a significantly smaller time step $(\Delta t=T / 400)$ than used
tpr $k h=2 \pi$. In comparison, we have recently shown (see Klahn et al. [11]) that for $k h=0.3$ the HOS method of
Dommermuth \& Yue [14] only allows stable time integration of waves with $H / H_{\text {max }} \lesssim 0.3$ if a comparable accuracy
is to be achieved. When seen in this light, the time integration of the present method may therefore be considered to
be quite robust indeed.

\section{Simulation of crescent waves}

As a first truly three-dimensional test, we here test the method's ability to simulate the formation of crescent waves. The crescent pattern occurs due to quintet instabilities of steep steady deep-water nonlinear wave trains, and the test hus indicates whether the method is capable of handling a three-dimensional case with a high degree of nonlinearity. Historically, the research into crescent waves can be traced back to the beginning of the 1980s starting with the work of Saffman \& Yuen [31]. Working with an equation valid for weakly nonlinear waves on deep water due to Zakharov [13], hey showed that for a given pair of numbers $(p, q)$, the branch of solutions corresponding to steady two-dimensional yaves would at some steepness bifurcate into solution branches corresponding to the steady two-dimensional waves Dems modulated by a wave of wave numbers $\left(k_{p}, k_{q}\right)=(1+p, q) k$ where $k=2 \pi / \lambda$ is the fundamental wave number f the steady two-dimensional wave train. Shortly thereafter, Meiron et al. [?] confirmed by numerical calculations hat this bifurcation also takes place in the unapproximated equations of potential flow theory, and $\mathrm{Su}$ [33] found experimentally that if steep enough, steady two-dimensional wave trains indeed develop a three-dimensional shape - milar to that predicted by Meiron et al. Denoting the patterns that repeat themselves every second, third and fourth vavelength of the original steady nonlinear wave by L2, L3 and L4, respectively, Su found that the steady nonlinear waves would develop the L2, L3 and L4 patterns in roughly $90 \%, 10 \%$ and $1 \%$ of the experiments. The explanation $\mathrm{r}$ this finding is generally accepted to be a combination of the facts that the waves in Su's experiments contained small random disturbances including a mixture of the unstable disturbances found by McLean [34], and that for the teepness considered by $\mathrm{Su}$, the disturbances leading to the L2 pattern have significantly larger rates of growth than the disturbances leading to the L3 and L4 patters. Although the theoretical understanding of the formation of crescent waves has been in place for about 40 years, it was first around the start of this millennium that numerical simulations $\mathrm{f}$ these waves based on the full potential flow equations appeared, and to the best of our knowledge, Xue et al. [35] were the first to achieve this milestone. Fuhrman et al. [36], Fructus et al. [19] and Xu \& Guyenne [29] soon followed meaning that several authors have now successfully simulated the phenomena.

In this work we will confine ourselves to the numerical simulation of the most unstable L2 pattern. To be able to compare our results to those of $\mathrm{Su}$ [33], we consider steady two-dimensional waves of steepness $H / \lambda=0.105$ and take the relative water depth to be $k h=2 \pi$. For these parameters McLean's analysis predicts that the wave numbers of the most unstable disturbance leading to the L2 pattern correspond to $(p, q)=(0.5,1.23)$ and we therefore initialize 
$\eta$ and $\Phi_{s}$ in our simulations as steady two-dimensional waves perturbed by

$$
\begin{aligned}
& \widetilde{\eta}(x, y)=\varepsilon H \sin \left(k_{p} x\right) \cos \left(k_{q} y\right) \\
& \widetilde{\Phi}_{s}(x, y)=\frac{\varepsilon H \sqrt{g}}{\left(k_{p}^{2}+k_{q}^{2}\right)^{1 / 4}} \cos \left(k_{p} x\right) \cos \left(k_{q} y\right) \exp \left(\left(k_{p}^{2}+k_{q}^{2}\right)^{1 / 2} \eta(x, y, 0)\right)
\end{aligned}
$$

which corresponds to the dominant Fourier modes of the unstable disturbance. In (29) $\varepsilon$ is some (in principal infinitely) small dimensionless number and $\eta(x, y, 0)$ is the surface elevation of the steady two-dimensional wave train. Moreover,

ve take $L_{x}=4 \lambda$ and $L_{y}=4 \pi / k_{q}$ such that the L2 pattern is repeated twice in both the $x$ and $y$ directions and we erform the simulations using $N_{x}=64, N_{y}=16, N_{s}=8$ and $\epsilon=10^{-9}$ (not to be confused with the amplitude of the perturbation, $\varepsilon$.) We employ artificial damping in our simulations using $\alpha=0.5$ and take $b=1.1 \times \max \{\eta(x, y, 0)\}$, here the maximum is taken over all grid points in the $(x, y)$-plane, giving a reduction in domain size by roughly $93 \%$. Finally we take the time step to be $\Delta t=T / 50$.

Qualitatively our results are in good agreement with the experiments of $\mathrm{Su}$ and the above mentioned numerical tudies. This point is illustrated by figure 9 which shows the evolution of the perturbed steady two-dimensional waves i-hto the L2 pattern for $\varepsilon=10^{-4}$. Like Xue et al. and Fuhrman et al. we have found that from a qualitative point of view, the value of $\varepsilon$ is only important for the time scale on which the crescent pattern forms by which we mean hat larger values of $\varepsilon$ lead to a similar shape but at an earlier time. On the other hand, we have found the value of $\varepsilon$ to be quite important from a quantitative point of view. In the literature it has become common practice to assess he accuracy of simulations of the L2 pattern by comparing the wavelength and wave height ratios $\lambda_{2} / \lambda_{1}, h_{11} / h_{12}$, $h_{11} / h_{21}$ and $h_{21} / h_{22}$ as well as the maximum steepness $\left(\max \partial_{x} \eta\right)$ all evaluated at $y=\pi / k_{q}$ to the experimental results f $\mathrm{Su}$ where the quantities $\lambda_{1}, \lambda_{2}, h_{11}, h_{12}, h_{21}$ and $h_{22}$ are defined in figure 10. Our results for the wavelength and wave height ratios are shown in figure 11 for different values of $\varepsilon$ up to the point in time where the damping changes the shape of the crescent pattern significantly, and we conclude that the local variation of the quantities is $O(\varepsilon)$. For that reason we find a comparison to Su's results most meaningful when $\varepsilon$ is chosen smaller than 0.01 , since his results are given with two digits after the decimal point. Table 4 shows our results for the above mentioned quantities as a function of time when using $\varepsilon=10^{-4}$, and it is clear that at no instant in time do we reach a perfect agreement with the results of $\mathrm{Su}$. On the other hand, we do get a reasonable agreement at e.g. $t=43 T$ where our results for $h_{11} / h_{12}$, $l_{21} / h_{22}, \lambda_{2} / \lambda_{1}$ and $h_{11} / h_{21}$ are about $1 \%, 1 \%, 12 \%$ and $9 \%$ off those of $\mathrm{Su}$, and our result for max $\partial_{x} \eta$ is about $32 \%$ and $16 \%$ off the result of $\mathrm{Su}$ depending on which value we compare to. We note that these deviations are similar to lose obtained in the simulations by Xue et al., Fuhrman et al. and Xu \& Guyenne, and on average somewhat larger than those obtained in the simulations by Fructus et al. who, however, used $H / \lambda=0.095$ as the initial steepness of the steady two-dimensional waves. It should be noted that for this steepness, the instability leading to the L2 pattern is not the strongest one and is in fact dominated by two-dimensional Benjamin-Feir type instabilities.

Another way to check the accuracy of the simulation is to compare the growth rate of the initial disturbance to drowth rate predicted by the analysis of McLean [34]. At any instant in time the free surface elevation may be written in the form (3a) and the amplitude of the initial disturbance can be directly measured by the absolute value the coefficient $\widehat{\eta}_{6,2}$ since the disturbance repeats itself six times in the $x$-direction and two times in the $y$-direction ver the chosen domain. The time evolution of $\left|\widehat{\eta}_{6,2}\right|$ when using the parameters $\varepsilon=10^{-4}, N_{x}=64, N_{y}=16, N_{s}=8$, $\epsilon=10^{-9}, \alpha=0.5$ and $\Delta t=T / 50$ is shown in figure 12 together with the prediction of McLean, namely, that $\left|\widehat{\eta}_{6,2}\right|$ proportional to $\exp (0.0316 \sqrt{g k} t)$. The results are seen to match well at all times, except at the very beginning of the simulation. This early discrepancy may be explained by the fact that the initial disturbance (29) corresponds to a

rinear combination of eigenfunctions of McLean rather than just the most unstable eigenfunction, and for such a linear ombination the time evolution will only resemble that of the most unstable eigenfunction for large enough times. Because the results of McLean in principle only predict the time evolution of the steady wave train immediately after has been perturbed, it is interesting to note that our results show that the disturbance grows at the same rate as that predicted by McLean at all times during the formation of the L2 pattern. This finding differs from the results of Fuhrman et al. [36], who found the growth to accelerate during the formation. At the present time we believe the peason for this disagreement to be that Fuhrman et al. measured the rate of growth differently than we have done in this work.

\section{Simulation of a nonlinear wave focusing event}

As a further validation of the method, we employ it to simulate a focusing event in an irregular wavefield. Our strategy for simulating such an event is to initialize the wavefield at time $t_{0}<0$ such that linear theory predicts the event to occur with maximum crest elevation $A$ at time $t=0$. This idea has previously been used in the experimental investigations of Baldock et al. [37] and Johannessen \& Swan [38] (which we will refer to as 'JS' throughout this 
(a)

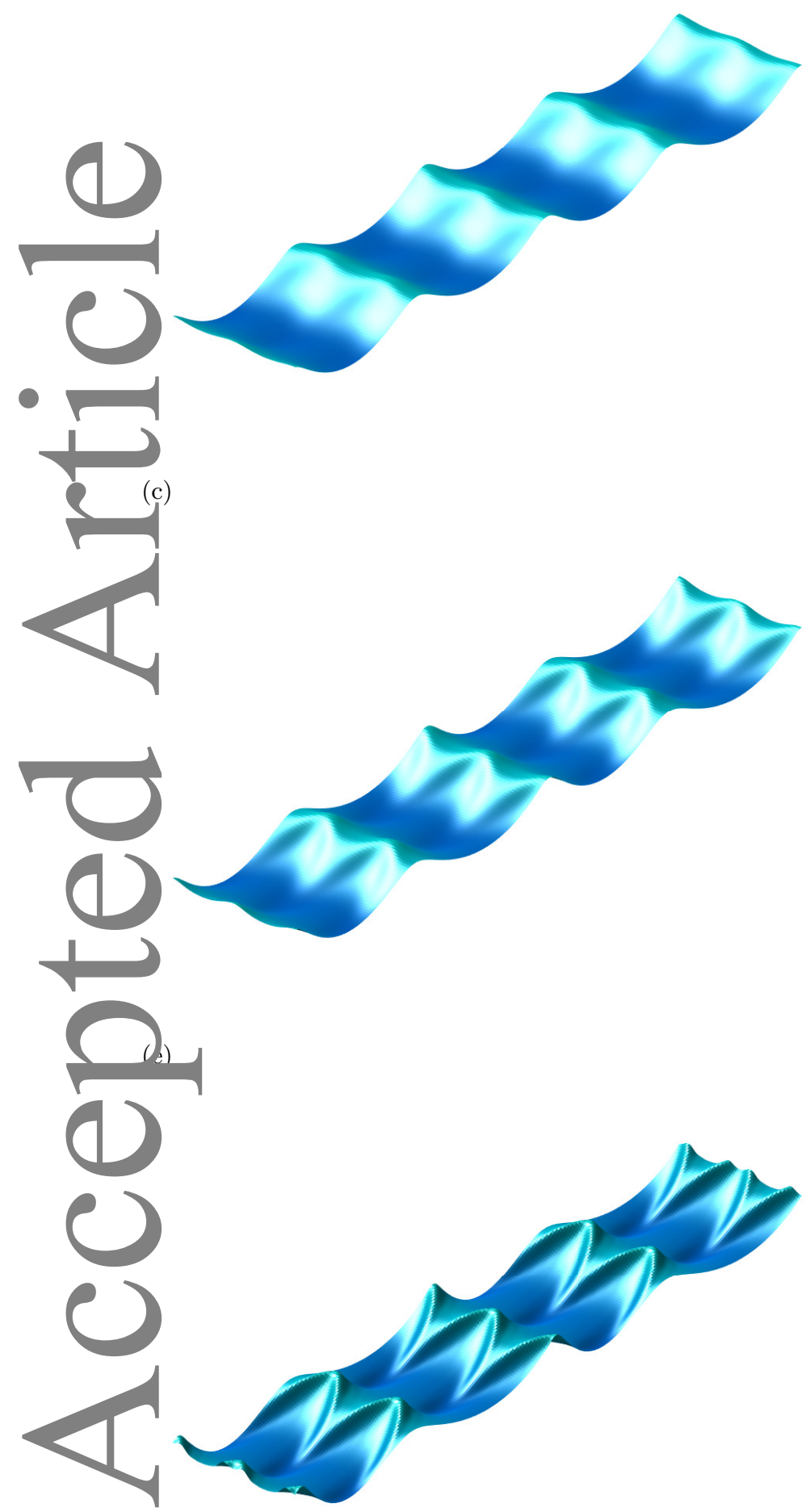

(b)

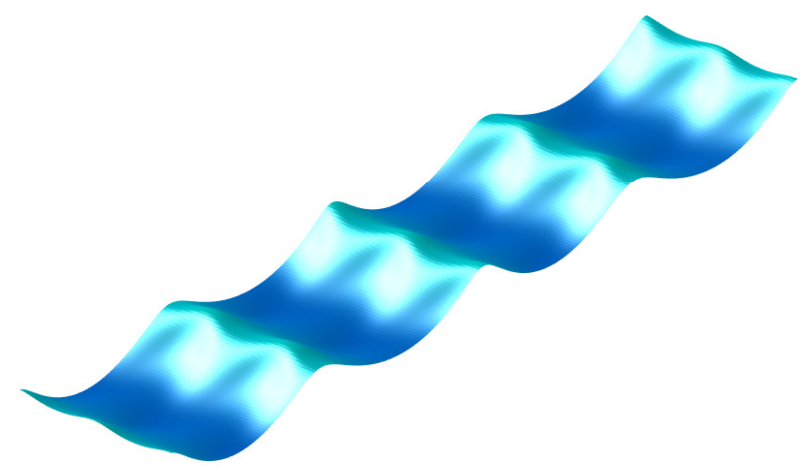

(d)

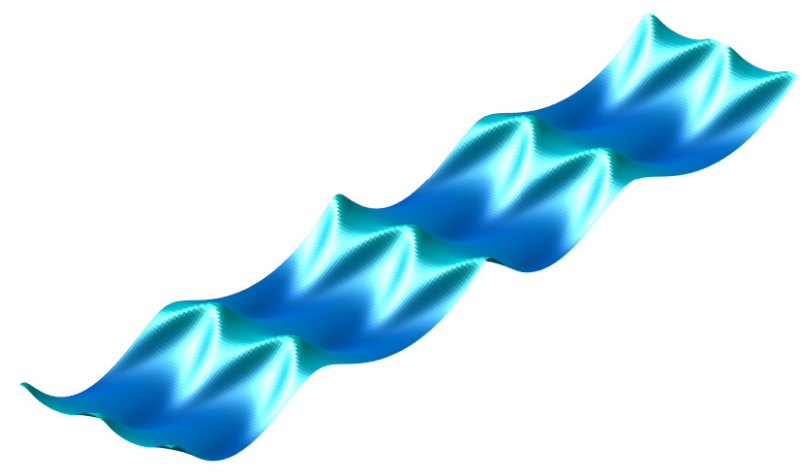

(f)

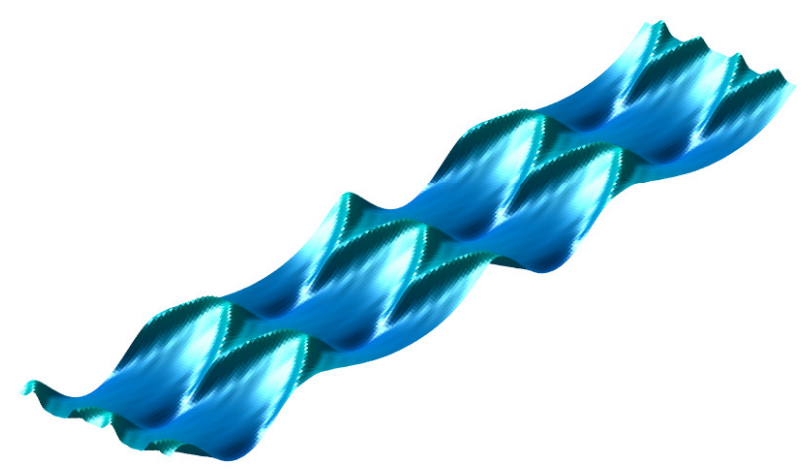

Figure 9: The formation of the L2 pattern over time. The figure shows the surface elevation at time (a) $t=37 T$, (b) $t=39 T$, (c) $t=41 T$, (d) $t=43 T$, (e) $t=45 T$ and (f) $t=47 T$ when using the parameters $N_{x}=64, N_{y}=16, N_{s}=8$, $\epsilon=10^{-9}, \alpha=0.5$ and $\Delta t=T / 50$. The amplitude of the initial perturbation is $\varepsilon=10^{-4}$. 


\begin{tabular}{|c|c|c|c|c|c|c|c|c|c|c|c|c|c|}
\hline \multirow[b]{2}{*}{$t / T$} & \multicolumn{12}{|c|}{ Present method } & \multirow[t]{2}{*}{$\mathrm{Su}[33]$} \\
\hline & 36 & 37 & 38 & 39 & 40 & 41 & 42 & 43 & 44 & 45 & 46 & 47 & \\
\hline$\lambda_{2} / \lambda_{1}$ & 1.05 & 1.06 & 1.06 & 1.08 & 1.09 & 1.12 & 1.14 & 1.16 & 1.19 & 1.21 & 1.21 & 1.20 & 1.28 \\
\hline$h_{11} / h_{12}$ & 1.03 & 1.04 & 1.04 & 1.05 & 1.06 & 1.07 & 1.09 & 1.11 & 1.14 & 1.18 & 1.25 & 1.34 & 1.10 \\
\hline$h_{11} / h_{21}$ & 1.10 & 1.13 & 1.15 & 1.19 & 1.23 & 1.29 & 1.36 & 1.45 & 1.57 & 1.71 & 1.80 & 1.80 & 1.66 \\
\hline$h_{21} / h_{22}$ & 0.97 & 0.96 & 0.96 & 0.95 & 0.93 & 0.92 & 0.90 & 0.87 & 0.84 & 0.79 & 0.73 & 0.69 & 0.88 \\
\hline $\max \partial_{x} \eta$ & 0.58 & 0.60 & 0.62 & 0.64 & 0.68 & 0.72 & 0.78 & 0.86 & 0.95 & 1.03 & 0.94 & 0.64 & $0.65,1.02$ \\
\hline
\end{tabular}

(1)

able 4: The values of the wavelength and wave height ratios as a function of time computed with the parameters ${ }_{x}=64, N_{y}=16, N_{s}=8, \epsilon=10^{-9}, \alpha=0.5, \Delta t=T / 50$ and $\varepsilon=10^{-4}$ together with the values of $\mathrm{Su}$ [33].
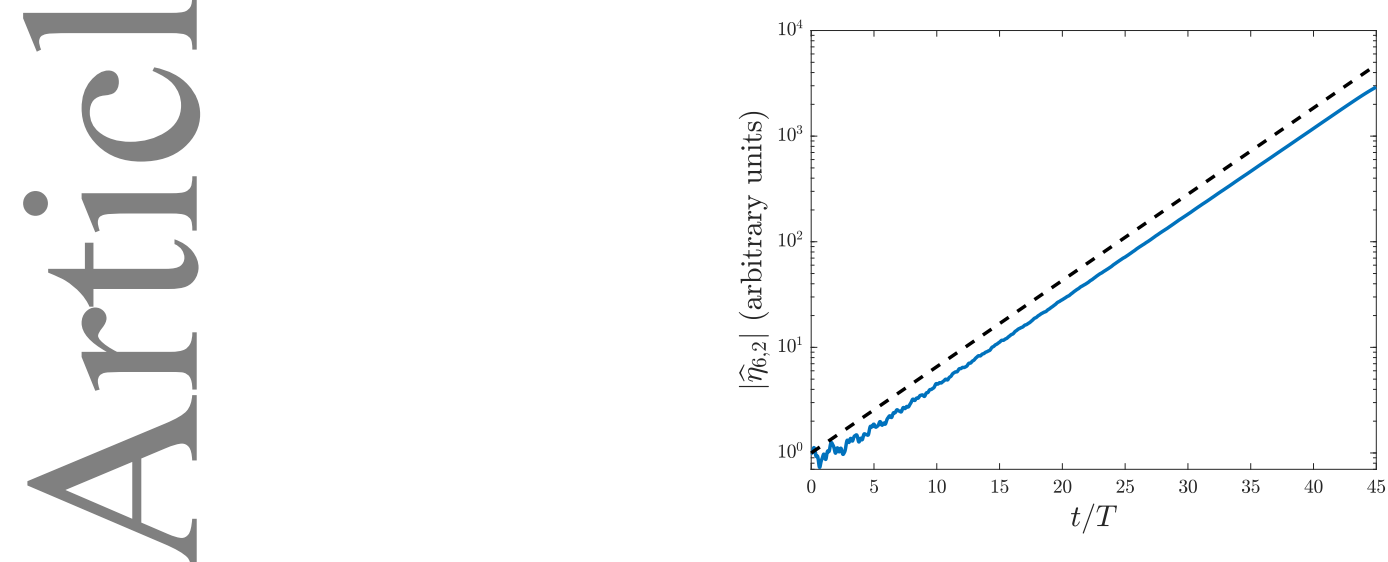

Figure 12: The time evolution of $\left|\widehat{\eta}_{6,2}\right|$ as a function of time when using the parameters $\varepsilon=10^{-4}, N_{x}=64, N_{y}=16$, $N_{s}=8, \epsilon=10^{-9}, \alpha=0.5$ and $\Delta t=T / 50$. The dashed line is given by $\exp (0.0316 \sqrt{g k} t)$ and corresponds to the volution predicted by McLean's analysis.

Tection) as well as in the numerical studies of Bateman et al. [39] and Fuhrman \& Madsen [40]. While the mentioned athors all studied a wide variety of focusing events, we will here confine ourselves to simulating the case denoted as B78s4 by JS. For this case $A=78 \mathrm{~mm}$ while the frequency, $\omega$, and angle of propagation, $\theta$ (measured counter-clockwise om the $x$-axis), of the initial wave field are distributed according to the distribution $\mu(\omega, \theta)=\chi(\omega) \Gamma(\theta)$, where

$\chi(\omega) \propto \begin{cases}\omega^{-2} & \text { if } \omega_{\min } \leq \omega \leq \omega_{\max } \\ 0 & \text { otherwise }\end{cases}$

(1)

$\Gamma(\theta) \propto \begin{cases}\cos \left(\frac{\theta}{2}\right)^{4} & \text { if } \theta_{\min } \leq \theta \leq \theta_{\max } \\ 0 & \text { otherwise. }\end{cases}$

the above equations $\omega_{\min }=92 \pi / 64 \mathrm{rad} / \mathrm{s}, \omega_{\max }=212 \pi / 64 \mathrm{rad} / \mathrm{s}, \theta_{\min }=-\pi / 4 \mathrm{rad}$ and $\theta_{\max }=\pi / 4 \mathrm{rad}$. The platural starting point for our numerical method is a wave number spectrum rather than a frequency-direction spectrum, and for that reason we convert $\mu(\omega, \theta)$ to the corresponding wave number spectrum, $\rho(\mathbf{k})$, where $\mathbf{k}$ is the vector of length $\kappa$ and angle $\theta$ relative to the $x$-axis. To do the conversion, we assume that $\rho(\mathbf{k})=\psi(\kappa) \Gamma(\theta)$ and that $\omega$ and $\mathbf{k}$ are related through the linear dispersion relation $\omega(\mathbf{k})^{2}=g \kappa \tanh (\kappa h)$. Using the fact that for all values of $\kappa_{1}, \kappa_{2}, \theta_{1}$ and $\theta_{2}$ the energy contained in the wave components with wave vectors in the set $\left\{\mathbf{k} \mid \kappa_{1} \leq \kappa \leq \kappa_{2}\right.$ and $\left.\theta_{1} \leq \theta \leq \theta_{2}\right\}$ is the same as the energy contained in the wave components with frequencies and propagation angles in the set $\left\{(\omega, \theta) \mid \omega\left(\kappa_{1}\right) \leq \omega \leq \omega\left(\kappa_{2}\right)\right.$ and $\left.\theta_{1} \leq \theta \leq \theta_{2}\right\}$, it can be shown that $\chi(\omega)$ and $\psi(\kappa)$ are connected through the relation (see for example Chapter 2 in the book by Young [28])

$$
\psi(\kappa)=\frac{1}{\kappa} \frac{\mathrm{d} \omega}{\mathrm{d} \kappa} \chi(\omega(\kappa))
$$




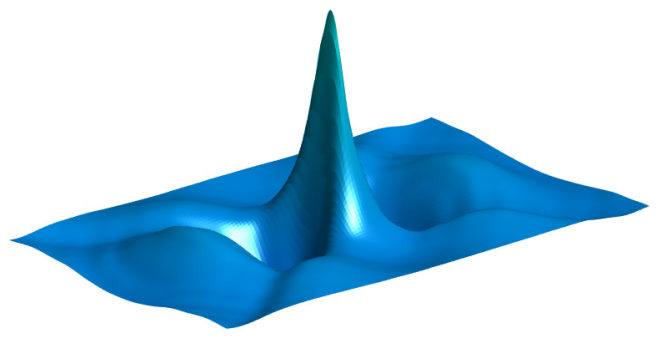

igure 13: A zoom-in on the surface elevation at the focus time computed using the parameters $L_{x}=L_{y}=25.6 \mathrm{~m}$, $h=1.2 \mathrm{~m}, A=78 \mathrm{~mm}, t_{0}=-20 \mathrm{~s}, \Delta t=0.01 \mathrm{~s}, N_{x}=N_{y}=128, N_{s}=14, b=0.12 \mathrm{~m}, \epsilon=10^{-9}$ and $\alpha=0.7$.

Implying that the total wave number spectrum is given by

$$
\rho(\mathbf{k}) \propto \begin{cases}\frac{1}{\kappa \omega(\kappa)^{2}} \frac{\mathrm{d} \omega}{\mathrm{d} \kappa} \cos \left(\frac{\theta}{2}\right)^{4} & \text { if } \kappa_{\min } \leq \kappa \leq \kappa_{\max } \text { and } \theta_{\min } \leq \theta \leq \theta_{\max } \\ 0 & \text { otherwise. }\end{cases}
$$

rom this distribution we choose the Fourier coefficients of $\eta$ at time $t=0$ such that they are all positive numbers and add up to $A$. From $\eta$ at time $t=0$ we obtain $\eta$ at time $t=t_{0}$ by using linear theory, and we obtain $\Phi_{s}$ at $t_{0}$ utilizing the relation between the Fourier coefficients of $\Phi_{s}$ and $\eta$ from linear theory.

Employing this procedure for initializing $\eta$ and $\Phi_{s}$ at $t_{0}=-20 \mathrm{~s}$, we have simulated the B78s 4 case of JS using the arameters $L_{x}=L_{y}=25.6 \mathrm{~m}, h=1.2 \mathrm{~m}, A=78 \mathrm{~mm}, \Delta t=0.01 \mathrm{~s}, N_{x}=N_{y}=128, N_{s}=14, b=0.12 \mathrm{~m}$ (roughly corresponding to reduction of the domain size by $90 \%), \epsilon=10^{-9}$ and $\alpha=0.7$. In our simulations the focus occurs at he time $t_{f}=0.15 \mathrm{~s}$ (relative to $t_{f}=0$ predicted by linear theory) and the surface elevation at that time is shown in gure 13. Moreover, our results for the time evolution of the surface elevation at the focus point as well as the vertical profile of the velocity in the $x$-direction, $u=\partial_{x} \Phi$, at the focus point and time are shown in figure 14 together with the xperimental results of JS. Although the numerical results cannot be expected to match the experimental results of JS perfectly, since the initial condition of the simulation necessarily differs from that used in the experimental wave tank, the overall agreement is very good indeed. To quantify the deviation, we here note that our result for the maximum ce elevation is about $1.8 \%$ smaller than that of JS. Our results for the velocity profile are also in good agreement

with the results of JS, and we note that the present numerical method avoids the small oscillations in the computed elocity profile near the seabed of the Boussinesq-type model used by Fuhrman \& Madsen [40].

\section{Simulation of a Gaussian hump initially at rest}

As a final test of the method, we use it to simulate the time evolution of a Gaussian hump which is initially at rest. esting the quality of the solution obviously requires a reference solution, and to the best of our knowledge, there is no (numerically) exact solution to the nonlinear case. In the limit of small height-to-width-ratio of the hump, the time volution is, however, going to be predicted by the linear equations obtained from (1) by linearizing around the rest state, and this gives us the opportunity to test the numerical method. Aside from testing the method in this limit, we also present results for a nonlinear case with the aim of providing a reference solution for future tests.

To carry out the simulation we let $L_{x}=L_{y}=L$ where $L=1 \mathrm{~m}$ and denote the initial height and characteristic width of the hump by $H$ and $\sigma$, respectively. We take the initial surface elevation at the $\left(n_{x}, n_{y}\right)$ th grid point to be

$$
\eta\left(x_{n_{x}}, y_{n_{y}}, 0\right)=H \exp \left(-\frac{\left(x_{n_{x}}-L / 2\right)^{2}+\left(y_{n_{y}}-L / 2\right)^{2}}{\sigma^{2}}\right)
$$

and we take the initial surface potential, $\Phi_{s}(x, y, 0)$, to be a constant over the domain. It is clear that the initial condition for $\eta$ is not periodic in $x$ and $y$, but we note here, that it becomes an exponentially accurate approximation to a periodic function in the limit where $\sigma / L$ is small. Denoting the $\left(n_{x}, n_{y}\right)$ th Fourier coefficient of $\eta(x, y, 0)$ by $\widehat{\eta}_{n_{x}, n_{y}}$ 
(a)

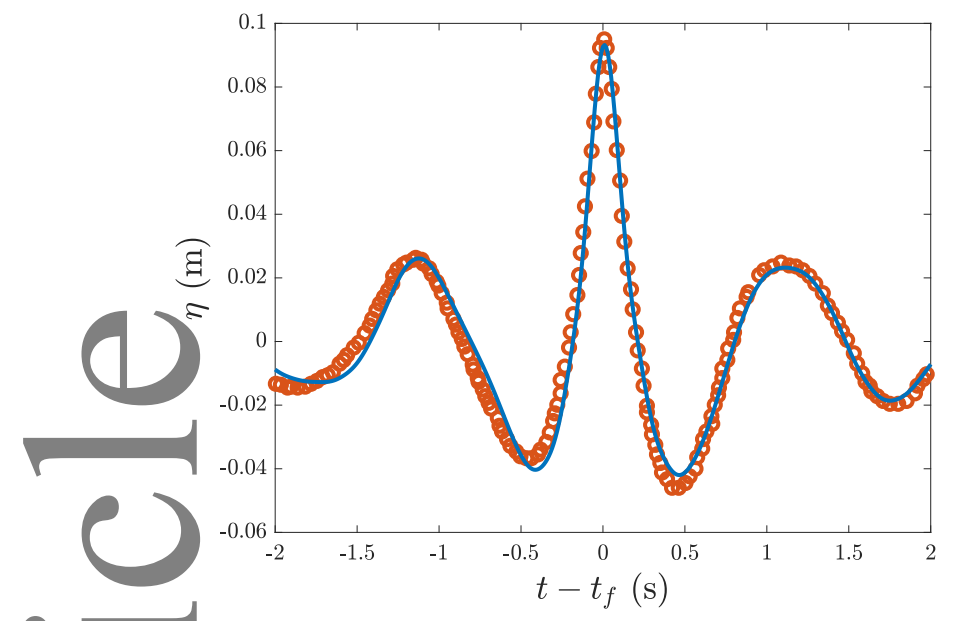

(b)

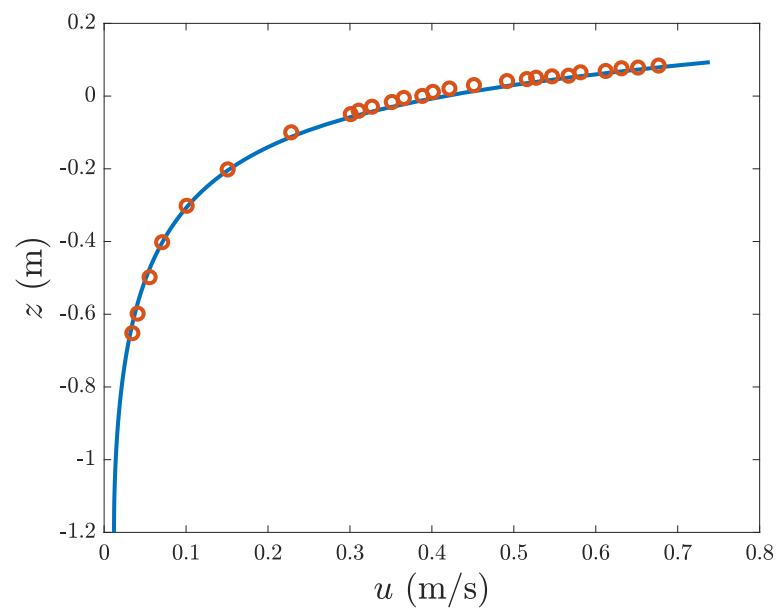

Figure 14: (a): The surface elevation at the focus point as a function of time relative to the focusing to the time of cus $t_{f}$. (b): The vertical profile of the velocity in the $x$-direction at the focus point and time. Our results have been obtained using the parameters $L_{x}=L_{y}=25.6 \mathrm{~m}, h=1.2 \mathrm{~m}, A=78 \mathrm{~mm}, t_{0}=-20 \mathrm{~s}, \Delta t=0.01 \mathrm{~s}, N_{x}=N_{y}=128$, $T_{s}=14, b=0.12 \mathrm{~m}, \epsilon=10^{-9}$ and $\alpha=0.7$ and are shown as the blue line in both figures. The red circles are the results of Johannessen \& Swan [38].

if follows that the linearization of (1) predicts the surface elevation at time $t$ to be given by

$$
\eta^{(L)}(x, y, t)=\sum_{n_{x}=-N_{x}}^{N_{x}-1} \sum_{n_{y}=-N_{y}}^{N_{y}-1}\left(\widehat{\eta}_{n_{x}, n_{y}} \cos \left(\omega_{n_{x}, n_{y}} t\right)\right) \exp \left(i\left(\mathbf{k}_{n_{x}, n_{y}} \cdot \mathbf{r}\right)\right)
$$

where $\omega_{n_{x}, n_{y}}^{2}=g \kappa_{n_{x}, n_{y}} \tanh \left(\kappa_{n_{x}, n_{y}} h\right)$ which is the solution we test against in the limit of small $H / \sigma$. Based on the rder of magnitude estimates given by Lautrup [41] (see Section 24 in his book) we measure time in units of $\sigma / \sqrt{g h}$ nd we choose the time step as

$$
\Delta t=C \frac{\sigma}{\sqrt{g h}}
$$

ith $C \leq 1$ a dimensionless number. We take $b=H$ which in all cases will give a reduction in domain size by about $94 \%$ or more and we perform the simulations in this section without any artificial damping.

\section{.1 Convergence to the result of linear theory in the limit $H / \sigma \rightarrow 0$}

o test the convergence of our numerical solution to (35) we consider a Gaussian hump with $h / L=1$ and $\sigma / L=0.02$ and use the computational parameters $N_{x}=N_{y}=16, N_{s}=6, C=1 / 10$ and $\epsilon=10^{-14}$ to integrate the system in time until $t_{f}=25 \sigma / \sqrt{g h}$. At this time we compute the deviation between the simulated result and the result of linear leory as

$$
\operatorname{Deviation}[\eta]=\sqrt{\frac{\sum_{n_{x}=0}^{2 N_{x}-1} \sum_{n_{y}=0}^{2 N_{y}-1}\left|\eta\left(x_{n_{x}}, y_{n_{y}}, t_{f}\right)-\eta^{(L)}\left(x_{n_{x}}, y_{n_{y}}, t_{f}\right)\right|^{2}}{\sum_{n_{x}=0}^{2 N_{x}-1} \sum_{n_{y}=0}^{2 N_{y}-1}\left|\eta^{(L)}\left(x_{n_{x}}, y_{n_{y}}, t_{f}\right)\right|^{2}}} .
$$

To steer clear of any possible misconceptions, we stress that Deviation $[\eta]$ does not directly measure the error of the numerical simulations since we do not compare to the exact solution but only to the solution of the linearized problem. Our results for Deviation $[\eta]$ as a function of $H / \sigma$ are given in figure 15 and it is clear to see that the deviation increases linearly with $H / \sigma$ as long as $H / \sigma \geq 10^{-6}$. The fact that the deviation decreases with $H / \sigma$ for $H / \sigma<10^{-6}$ we ascribe to rounding errors, for when computing the absolute deviation (i.e. (37) with a denominator of 1 ), we have found that the deviation stagnates for $H / \sigma<10^{-6}$. As such we are confident that our model works as intended. 


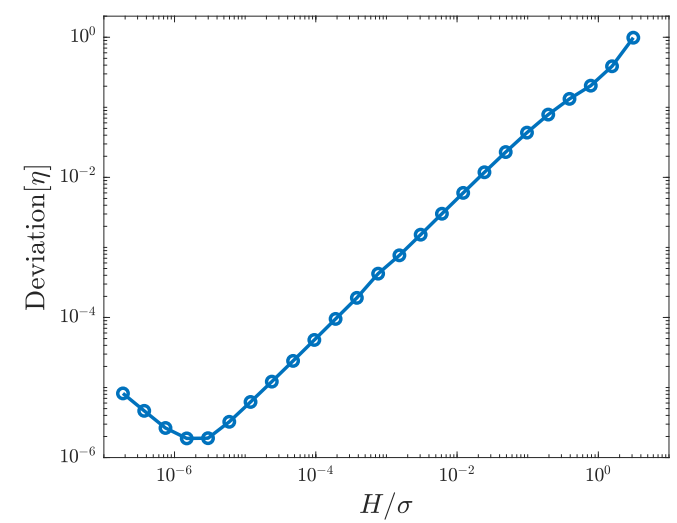

igure 15: Deviation[ $\eta]$ as a function of $H / \sigma$ for the case $h / L=1$ and $\sigma / L=0.02$. The computational parameters sed to produce the figure are $N_{x}=N_{y}=16, N_{s}=6, C=1 / 10$ and $\epsilon=10^{-14}$.
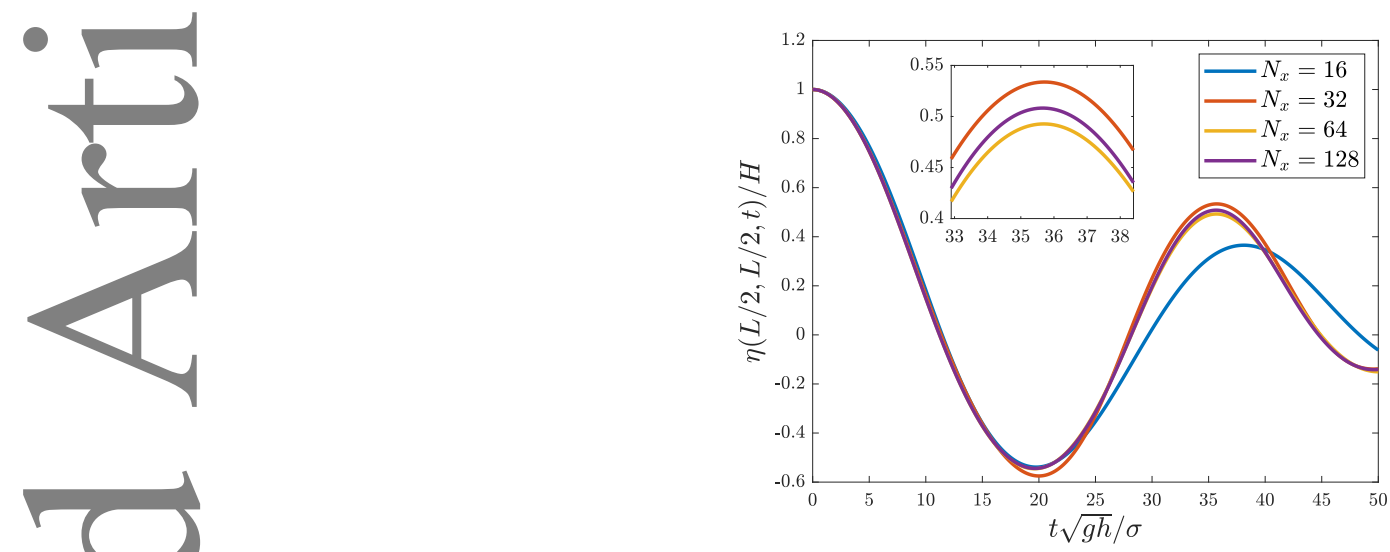

Figure 16: The surface elevation at $(x, y)=(L / 2, L / 2)$ as a function of time when using the parameters $h / L=1$, $L=0.02, H / L=2^{-6}, N_{y}=N_{x}, N_{s}=14, C=0.1$ and $\epsilon=10^{-14}$.

\section{.2 A nonlinear benchmark result}

Irom the results shown in figure 15 it can be seen that the prediction of linear theory is off by roughly $20 \%$ at $t=t_{f}$ f ${ }^{2} / / L=2^{-6}$ (corresponding to $H / \sigma=0.78$ ) when $h / L=1$ and $\sigma / L=0.02$. In an attempt to find the exact, nonlinear behavior of this case, we will in this section study its convergence as a function of the horizontal resolution sing the computational parameters $N_{s}=14, C=1 / 10$ and $\epsilon=10^{-14}$. The quantity, which we use to measure the onvergence, is the surface elevation at the domain center, $\left(x_{c}, y_{c}\right)=(L / 2, L / 2)$.

The surface elevation at $\left(x_{c}, y_{c}\right)$ is shown as a function of time in figure 16 for different values of $N_{x}=N_{y}$. As xpected, the surface elevation oscillates up and down in time and it is seen that the curves come closer together as $N_{x}$ increases. To quantify the convergence, the values of $\eta(L / 2, L / 2, t)$ at the times $t \sqrt{g h} / \sigma=10,20,30,40$ and 50 re given in table 5 as a function of $N_{x}=N_{y}$. For a fixed time, the surface elevation converges to some value although he rate of convergence is clearly larger for small $t$ than for large $t$. One might of course wonder why so fine resolution is needed in order to obtain accurate results for this case. For that reason we show a zoom-in on the non-zero part f the free surface elevation at the times $t \sqrt{g h} / \sigma=10,20,30,40$ and 50 in figure 17 which illustrates the fact that $\eta(x, y, t)$ locally exhibits large spatial variations and thereby explains the need for fine resolution.

\section{Computational cost}

Having shown that the present numerical method is capable of accurately simulating a number of nonlinear wave phenomena, we use this section to discuss its computational cost. We first derive the expected scaling of the computational cost in terms of the parameters $N_{x}, N_{y}$ and $N_{s}$, before we report on the actual CPU time per time step required to run the simulations. To put these results into perspective we mention here that the numerical method has been programmed in Matlab R2019a and executed on a HP Elitebook laptop with 16 GB RAM and an Intel i5-8250U 1.6 $\mathrm{GHz}$ processor. 


\begin{tabular}{|c|c|c|c|c|c|}
\hline \multirow[b]{2}{*}{$N_{x}$} & \multicolumn{5}{|c|}{$t \sqrt{g h} / \sigma$} \\
\hline & 10 & 20 & 30 & 40 & 50 \\
\hline 16 & 0.182547 & $-\mathbf{0 . 5 3 8 5 3 4}$ & 0.022585 & $\mathbf{0 . 3 4 6 6 3 0}$ & -0.062052 \\
\hline 32 & 0.163578 & $-\mathbf{0 . 5 7 4 9 7 6}$ & $\mathbf{0 . 2 3 0 3 6 2}$ & $\mathbf{0 . 3 7 0 3 3 0}$ & $-\mathbf{0 . 1 3} 8438$ \\
\hline 48 & 0.147206 & -0.538029 & 0.188467 & 0.350112 & $-\mathbf{0 . 1 4 5 4 9 1}$ \\
\hline 64 & 0.149869 & $-\mathbf{0 . 5 4 4 3 0 1}$ & 0.190157 & $\mathbf{0 . 3 3 5 0 2 1}$ & $-\mathbf{0 . 1 5 0 3 8 7}$ \\
\hline 96 & 0.149139 & $-\mathbf{0 . 5 4 3 1 7 4}$ & 0.195174 & $\mathbf{0 . 3 3 6 8 1 9}$ & $-\mathbf{0 . 1 4 3 9 8 7}$ \\
\hline 128 & 0.149069 & -0.543270 & 0.195443 & 0.336397 & $-\mathbf{0 . 1 4 0 9 6 0}$ \\
\hline 192 & 0.149062 & -0.543283 & 0.195385 & $\mathbf{0 . 3 3 6 4 2 2}$ & $-\mathbf{0 . 1 3 9 6 9 3}$ \\
\hline 256 & 0.149062 & -0.543283 & 0.195379 & 0.336397 & -0.139814 \\
\hline
\end{tabular}

Table 5: The values of $\eta(L / 2, L / 2, t) / H$ for different values of $N_{x}$ and $t$ when using the parameters $h / L=1, \sigma / L=0.02$, $Y / L=2^{-6}, N_{y}=N_{x}, N_{s}=14, C=0.1$ and $\epsilon=10^{-14}$. The bold digits indicate those matching the result obtained ith $N_{x}=256$.

\subsection{Expected computational cost}

In order to advance $\eta$ and $\Phi_{s}$ a single time step, the method in short performs three tasks: It solves the transformed aplace equation (15), computes $w_{s}$ using (23) and finally evaluates the right hand sides of (1c) and (1d). Of these, the computational cost of the first task overwhelms the cost of the latter two, and the computational cost associated ith a single time step can thus be analyzed by considering the computational cost of solving (15).

Now, when using the preconditioning strategy outlined in Section 3.2.3 we have found that the number of GMRES iterations needed to solve (15) for all practical purposes is bounded by a constant, say, 40. As such, the computational complexity of the method must be the same as the computational complexity of a single iteration, which we recall consists of applying the preconditioner and evaluating the right hand side of (15). The cost associated with applying the preconditioner is dominated by the inversion of the matrices $\mathcal{L}$ and $\mathcal{U}$, whose sparsity pattern is shown in Figure Since these matrices both have $N_{s}$ bands of non-zero entries with a width of one, it is possible to invert them sing e.g. Gaussian elimination in at most $O\left(N_{x} N_{y} N_{s}^{2}\right)$ operations. The cost associated with evaluating the right hand side of (15) is dominated by the computation of the derivatives of $F$, because the pointwise multiplication of the erivatives with their coefficients has a cost which scales linearly with $N_{x}, N_{y}$ and $N_{z}$. The computation of $x$-derivatives requires $O\left(N_{y} N_{s}\right)$ fast Fourier transforms of vectors of length $O\left(N_{x}\right)$, meaning that the total computational complexity ecomes $O\left(N_{x} \log \left(N_{x}\right) N_{y} N_{s}\right)$. Using a similar argument one may show that the computation of $y$-derivatives requires $O\left(N_{x} N_{y} \log \left(N_{y}\right) N_{s}\right)$ operations in total. To compute the $s$-derivatives of $F, O\left(N_{x} N_{y}\right)$ matrix vector products with opmplexity $O\left(N_{s}^{2}\right)$ are needed, since the differentiation matrices in the $s$-direction are dense. From this it follows that the domputational cost associated with the computation of the $s$-derivatives is $O\left(N_{x} N_{y} N_{s}^{2}\right)$. In total we therefore conclude that a single iterations requires $O\left(N_{x} \log \left(N_{x}\right) N_{y} N_{s}+N_{x} N_{y} \log \left(N_{y}\right) N_{s}+N_{x} N_{y} N_{s}^{2}\right)$ operations.

Denoting the total number of grid points as $N=4 N_{x} N_{y}\left(N_{s}+1\right)$ we deduce the following from the above estimation: $N_{y}$ (or alternatively $N_{x}$ ) and $N_{s}$ are held constant while $N_{x}$ (or alternatively $N_{y}$ ) is, the computational cost of the method should be proportional to $N \log (N)$. If, on the other hand $N_{x}$ and $N_{y}$ are held constant while $N_{s}$ is varied, le computational cost of the method should be proportional to $N^{2}$. Finally, if only $N_{s}$ is held constant while $N_{x}$ and $N_{y}$ are both varied such that we always have $N_{x}=N_{y}$, the computational cost is expected to to be proportional to $\mathrm{V} \log (N)$.

\subsection{Actual computational cost}

To illustrate the actual computational cost of the numerical method we consider the cost associated with the simulation of the nonlinear benchmark result for the Gaussian hump initially at rest described in Section 7.2. To that end we gain use the parameters $h / L=1, \sigma / L=0.02, H / L=2^{-6}, N_{y}=N_{x}, C=0.1$ and $\epsilon=10^{-14}$ in connection with no artificial damping, and consider the CPU time per time step when keeping $N_{s}$ fixed while varying $N_{x}=N_{y}$ and when keeping $N_{x}$ fixed while varying $N_{s}$. The results of this computation are shown together with the expected computational cost in Figure 18, from which it may be seen that the actual cost matches the expected cost very well. We note, however, that only the first of the two cases is actually relevant for applications, since it is never necessary to choose large values of $N_{s}$ due to the artificial boundary condition limiting the vertical extent of the fluid domain.

It is of course interesting to compare the computational scaling of the present method to that of other methods. Engsig-Karup et al. [4] found the computational effort of their method to scale linearly with $N$ which as such appears more efficient than the present method. In that connection one should, however, keep in mind that the present method 


\section{Data availability statement}

Data have not been shared.

\section{Acknowledgement}

The authors gratefully acknowledge funding received from Centre for Oil and Gas - DTU/Danish Hydrocarbon Research and Technology Centre (DHRTC). Project name: DEWIOS. Project ID: FL_110.

\section{(1) References}

[1] TSAI, W.-T. \& Yue, D. K. P. 1996 Computation of nonlinear free-surface flows. Annu. Rev. Fluid Mech. 28, 249-278.

2] LI, B. \& FLEMING, C. A. 1997 A three dimensional multigrid model for fully nonlinear water waves. Coastal Engineering. 30, 235-258

[3] BINGHAM, H. B. \& ZHANG, H. 2007 On the accuracy of finite-difference solutions for nonlinear water waves. J. Eng. Math. 58, 211-228

[4] ENGSig-KARUP, A. P., BINGHAM, H. B. \& LINDBERG, O. 2009 An efficient flexible-order model for 3D nonlinear water waves. J. Comput. Phys. 228, 2100-2118.

[5] Christiansen, T. B., BIngham, H. B., ENGSig-KARUP, A. P., DUCROZET, G. \& FERRANT, P. 2013 Efficient Hybrid-Spectral Model for Fully Nonlinear Numerical Wave Tank. Proceedings of the AMSE 2013 32nd International Conference on Ocean, Offshore and Arctic Engineering OMAE.

[6] YATES, M. L. \& BENOIT, M. 2015 Accuracy and efficiency of two numerical methods of solving the potential flow problem for highly nonlinear and dispersive water waves. Int. J. Numer. Meth. Fluids. 77, 616-640

7] RAOUlt, C. , BENOIT, M. \& YATES, M. L. 2019 Development and validation of a 3D RBF-spectral model for coastal wave simulation. J. Comput. Phys. 378, 278-302.

8] YATES, M. L. \& BENOIT, M. 2015 Accuracy and efficiency of two numerical methods of solving the potential flow problem for highly nonlinear and dispersive water waves. Int. J. Numer. Methods Fluids 77, 616-640.

[9] ENGSIG-KARUP, A. P., ESKILSSON, C. \& BIGONI D. 2016 Stabilised nodal spectral element method for fully nonlinear water waves. J. Comput. Phys. 318, 1-21.

IU KLAHN, M., MADSEN, P. A. \& FUHRMAN, D. R. 2020 A new $\sigma$-transform baed Fourier-LegendreGalerkin model for nonlinear water waves. Accepted by Int. J. Numer. Meth. Fluids. Available at: https://files.dtu.dk/u/7aWFGHYlhR7_pYUa/Manuscript.pdf?I

11] KLAHN, M., MADSEN, P. A. \& FUHRMAN, D. R. 2020 On the accuracy and applicability of a new implicit Taylor method and the High Order Spectral method on steady nonlinear waves. Proc. Roy. Soc. A 476, 20200436.

Y12] NICHOLLS, D. P. 2011 Efficient enforcement of far-field boundary conditions in the Transformed Field Expansions method. J. Comput. Phys. 230, 8290-8303.

13] ZAKHAROV, V. E. 1968 Stability of periodic waves of finite amplitude on the surface of a deep fluid. J. Appl. Mech. Tech. Phys. 9:190-194.

[14] DOMMERMUTH, D. G. \& YUE, D. K. P. 1987 A high-order spectral method for the study of nonlinear gravity waves. J. Fluid Mech. 184, 267-288.

[15] WEST, B.J., BRUECKNER, K.A., JANDA, R.S., MILDER, D.M., MILTON, R.L. 1987 A new numerical method for surface hydrodynamics. J. Geophys. Res. 92 (C11), 11803-11824.

[16] DuCRozet, G., BINGHAM, H. B., ENGSIG-KARUP, A. P., BONNEFOY, F. \& FERRANT, P. 2012 A comparative study of two fast nonlinear free-surface water wave models. Int. J. Numer. Meth. Fluids. 77, 616-640.

[17] ENGSIG-KARUP, A. P., MADSEN, M. G. \& GLIMBER, S. L. 2012 A massively parallel GPU-accelerated model for analysis of fully nonlinear free surface waves. Int. J. Numer. Meth. Fluids. 70, 20-36. 
[18] LONGUET-Higgins, M. S. 1953 On the decrease of velocity with depth in an irrotational water wave. Proc. Camb. Phil. Soc. 49, 552-560.

[19] FRUCTUS, D., CLAMOND, D., GRUE, J. \& KRISTIANSEN, Ø. 2005 An efficient model for three-dimensional surface wave simulations Part I: Free space problems. J. Comput. Phys. 205, 665-685.

[20] HeSthaVEn, J. S. \& WARBURTON, T. 2008 Nodal Discontinuous Galerkin Methods. Springer.

[21] KOPRIVA, D. A. 2009 Implementing Spectral Methods for Partial Differential Equations. Springer.

T2] SAAD, Y. \& SCHULTZ, M. H. 1986 GMRES: A generalized minimal residual algorithm for solving nonsymmetric linear systems. SIAM J. Sci. Stat. Comput. 7, 856-869.

[3] FUHRMAN, D. R. \& BINGHAM, H. B. 2004 Numerical solutions of fully non-linear and highly dispersive Boussinesq equations in two horizontal dimensions. Int. J. Numer. Meth. Fluids 44, 231-255.

4] BATTJES, J. A. 1974 Surf similarity. Proceedings of the 14th Coastal Engineering Conference, Copenhagen, $A S C E, 466-480$.

[25] RIENECKER, M. M. \& FENTON, J. D. 1981 A Fourier approximation method for steady water waves. J. Fluid Mech. 104, 119-137.

[26] CLAMOND, D. \& DUTYKH, D. 2018 Accurate fast computation of steady two-dimensional surface gravity waves in arbitrary depth. J. Fluid Mech. 844, 491-518.

27] MADSEN, P. A., BINGHAM, H. B. \& LIU, H. 2002 A new Boussinesq method for fully nonlinear waves from shallow to deep water. J. Fluid Mech. 462, 1-30.

[28] YOUNG, I. R. 1999 Wind generated ocean waves. Elsevier.

[29] XU, L. \& GUYENNE, P. 2009 Numerical simulation of three-dimensional nonlinear water waves. J. Comput. Phys. 228, 8446-8466.

30] LONGUET-HIGGINS, M. S. \& COKELET, E. D. 1976 The deformation of steep surface waves on water I. A numerical method of computation. Proc. R. Soc. Lond. A. 350, 1-26.

31] SAFFMAN, P. \& YUEN, H. C. 1980 A new type of three-dimensional deep-water wave of permanent form. $J$. Fluid Mech. 101, 797-808.

[32] MEIRON, D. I., SAFFMAN, P. \& YUEN, H. C. 1982 Calculation of steady three-dimensional deep-water waves. J. Fluid Mech. 124, 109-121.

[33] SU, H.-Y. 1982 Three-dimensional deep-water waves. Part 1. Experimental measurement of skew and symmetric wave patterns. J. Fluid Mech. 124, 73-108.

[34] MCLEAN, J. W. 1982 Instabilities of finite-amplitude waves. J. Fluid Mech. 114, 315-330.

5] XUE, M., XÜ. H., LIU, Y. \& YUE, D. K. P. 2001 Computations of fully nonlinear three-dimensional wave-wave and wave-body interations. Part 1. Dynamics of steep three-dimensional waves. J. Fluid Mech. 438, 11-39.

6] Fuhrman, D. R., MADSEn, P. A. \& BInghaM, H. B. 2004 A numerical study of crescent waves. J. Fluid Mech. 513, 309-341.

[37] BALDOCK, T. E., SWAN, C. \& TAYLOR, P. H. 1996 A laboratory study of nonlinear surface waves on water. Proc. R. Soc. Lond. A 354, 649-676.

[8] JOHANNESSEN, T. B. \& SWAN, C. 2001 A laboratory study of the focusing of transient and directionally spread surface water waves. Proc. R. Soc. Lond. A 457, 971-1006.

[39] BATEMAN, W. J. D., SWAN, C. \& TAYLOR, P. H. 2001 On the Efficient Numerical Simulation of Directionally Spread Surface Water Waves. J. Comput. Phys. 174, 277-305.

[40] FUHRMAN, D. R. \& MADSEN, P. A. 2006 Numerical simulation of extreme events from focused directionally spread wavefields. Proceedings of the 30th International Conference on Coastal Engineering 1, 772-781.

[41] LAUTRUP, B. 2005 Physics of continuous matter. IOP Publishing. 